SOCIAL RESEARCH REPORTS

ISSN: 2066-6861 (print), ISSN: 2067-5941 (electronic)

\title{
GENDER ALCOHOL USE DISPARITIES AND (POSSIBLE) CONSEQUENCES ON MEN AND WOMEN HEALTH STATUS
}

\author{
Vlad Zeno MILLEA
}

Social Research Reports, 2019, Vol. 11, Issue 3, pp. 44-80

The online version of this article can be found at:

www. researchreports.ro

\section{https://doi.org/10.33788/srr11.3.4}

Published by:

Expert Projects Publishing House

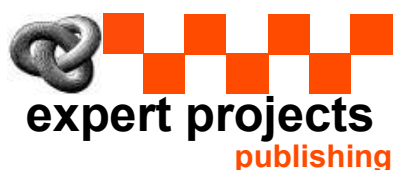

Covered by Index Copernicus International www.indexcopernicus.com

Directory of Open Access Journals

www.doaj.org

On behalf of:

Center for Program and Social Development

Aditional services and information about Social Research Reports can be found at:

www.researchreports.ro 


\title{
GENDER ALCOHOL USE DISPARITIES AND (POSSIBLE) CONSEQUENCES ON MEN AND WOMEN HEALTH STATUS
}

\author{
Vlad Zeno MILLEA ${ }^{1}$
}

\section{Abstract}

In this study we approached gender differences of alcohol consumption and, also, the way these differences are reflected in women and men harmful effects of drinking. We focused on Romanian evidences but we reported our results to European realities. The method was secondary data analysis, applied on information provided by World Health Organization, allowing us to create a European countries database, containing patterns of gendered consumption and some harmful consequences of drinking. Available data didn't allow us to analyze the important disparities between gendered patterns of alcohol consumption; so, we had to content us with some selection from scientific literature. Romania has a relatively high level of alcohol consumption but, also, an important proportion of abstainers and low consumers. The apparent contradiction could be explained by the high share of people with „heavy episodic drinking”. To note that women in Romania consume 4.2 times less alcohol than men, have a proportion of abstinence 3.1times higher and the percentage of those who drink daily or weekly is 6.1 times less than of men. Regarding the consequences of alcohol consumption, its dependence prevalence has a very low level in Romania but men have a 3.3 times higher risks. „Alcohol use disorder” has, again, a low prevalence but males have a 3.2 higher probability of AUD. Finally, we mention that in Romania there is an important proportion of deaths attributable to alcohol (for men the proportion is 2.6 higher than for women). An important finding: as much as global alcohol consumption increases, most of gender differences diminish.

Keywords: gender differences, patterns of alcohol consumption, alcohol use disorder, addiction, alcohol gender gap, antisocial.

\footnotetext{
${ }^{1}$ University "1 Decembrie 1918" from Alba Iulia, Faculty of Law and Social Science, Department of Social Science, Alba Iulia, Street: Unirii nr. 15-17, postal code: 510009, Alba Iulia, ROMANIA. E-mail: vladmillea@yahoo.com
} 


\section{Introduction}

We will start our analytic demarche presenting some aspects revealed by the investigations conducted on alcohol topic by World Health Organization in 2016, representing the most recent data made public by this organization. We intent to remind what a huge problem remains the consequences of alcohol consumption around the World and in Europe, particularly, and, also, to highlight which are the differences between men and women by this point of view.

The follow step of our introductive demarche consists in the presentation of some theories and scientific evidence targeting to explain gender gaps regarding alcohol consumption and its harmful consequences. We must note that available data did not contained information which make possible to analyze motives for gender gap in alcohol consumption patterns. We made a selective review of this kind of scientific literature in order to extend the theoretical debate proposed by our study and to create so a better background for further articles targeting gender disparities in alcohol consumption and its consequences.

\section{Alcohol consumption for men and women in 2016 revealed by World Health Organization}

From a recent paper, "Global status report on alcohol and health (GSRAH) 2018" we can find that in 2016 alcohol misuse generated 3.000.000 deaths (stand for $5.3 \%$ of all) and 132.6 million disability-adjusted life years, which represents $5.1 \%$ from the total of the year 2016. It's important to know that mortality due to alcohol abuse is superior to that generated by illness as tuberculosis, HIV/AIDS or diabetes. Analyzing separately by sex, the situation proves to be much more serious for men: 2.3 million deceased due to alcohol out of an entire of 3 million and 106.5 million disability-adjusted life years (also, generated by alcohol abuse) from the total of 132.6 million, counted in 2016. The same report reveals the presence of important disparities between men and women regarding last year prevalence of alcohol use disorders. At global level, 237 million men and 46 million women suffer of alcohol use disorders. The prevalence of this medical condition is highest in Europe (14.8\% for men and 3.5\% for women) and in the Region of Americas ( $11.5 \%$ for men and $5.1 \%$ for women).

Analyzing the "Percentage of total deaths attributable to alcohol, by age group, 2016" we can see that between 25 and 44 years old the proportion of deaths attributable to alcohol is highest all over the world and that Europe lies clearly on the first place. Gender gap (for people being 25-44 years old) is important: for men the proportion of deaths due to alcohol vary, approximately, between $31 \%$ and $29 \%$ and for woman, between $18 \%$ and $17 \%$. Concerning the specific causes of death, for each kind of illness or injury (intentional or unintentional) men have a larger absolute number of deaths than women and a bigger proportion of deceases attributable to alcohol (within each different medical condition). If we 
consider disability-adjusted life years caused by alcohol-attributable diseases, we find, again, that for every medical condition, the number of lost years is bigger for men than for women. The report shows, also, that, for each medical disorder, the rapport between the years lost due to alcohol and the sum of years lost because of respective illness (injury), is higher for males than for females. There is, also, an important gender difference regarding "alcohol use disorder": in Europe (which stands again on the world first place), $8.4 \%$ of men are alcohol addicted and $6.1 \%$ of them drink in harmful way. Regarding women, only $1.5 \%$ of them are alcoholic and $2 \%$ use alcohol in a damaging manner.

According to the authors (GSRAH, 2018), gender distribution of diseases and injuries alcohol-attributable comes, firstly, from the fact that men drink more than women. In addition, men have a greater risk of being affected by diseases and consequences of alcohol consumption because they engage more often in heavy alcohol consumption (including binge drinking) and practice risk behaviors related to drinking misuse, as smoking and use of tobacco products.

Some important differences between men's and women's alcohol patterns of consumption are revealed in "Global status report on alcohol and health": Europe has a low proportion of lifetime abstainers (second place after American regions) with $17.2 \%$ for males and $29.3 \%$ for females. Former drinkers represent $24.1 \%$ for males and $33.7 \%$ for females while the Current drinkers constitute $69.2 \%$ within male and $51.4 \%$ within female. With respect to alcohol consumption per capita (in liters of pure substance), Europe is characterized by a high level of this indicator, occupying the world second place, very close to the WHO Eastern Mediterranean Region. Men consumed, on average, 23.8 liters of pure alcohol but women drank only 8.3. In addition, in Europe the prevalence of heavy episodic drinking (among current drinkers) is the second of the world (after WHO African Region). The difference between genders is, again, important: for men the prevalence of heavy episodic drinking is $56.5 \%$ and for women, only $24.5 \%$.Regarding adolescents' "heavy episodic drinking", Europe occupy first place in the world, having a prevalence of $36.2 \%$ for boys and of $11.5 \%$ for girls. By longitudinal perspective, the Report (GSRAH, 2018) reveals, between 2000 and 2016, both sexes prevalence rates (in \%) for current alcohol consumers. In each year European male and female had the highest rates of current consumers but the trend is descendent for each of two genders (men descend from $78.8 \%$ to $69.2 \%$ and women, from $62.1 \%$ to $51.4 \%$ ). Final information on gender gap alcohol consumption offered by WHO that we presented, refers simultaneously to the relation between alcohol consumption, income and sex. Both for men and for women, the proportion of current drinkers decreases as the income become lower and, at each economic stands, the proportion of women current drinkers is significantly lower than men. For the total alcohol per capita consumption, the pattern is similar to those of current drinkers, with one exception: highest consumption corresponds to "upper middle income" and the second, to people with "high income". Regarding the Prevalence of Heavy Episodic Drinking (within drinkers), the situation is quite different: according to the prevalence of this behavior, those with low income lies on first place, followed by people having Upper middle income, then by person having High income and, finally, by those with lower middle income. It is interesting, also, to reveal that the ratio between the proportions of males and females which are current drinkers 
increases constantly as income becomes bigger while the ratio between men' and women' total consumption of alcohol remains quasi-constant regardless of income. The difference between women and men regarding the prevalence of heavy episodic drinking is the lowest for the category "low income" and is approximately constant for the others categories of revenue. As we already mentioned, in our study we are interested in gender gaps of alcohol consumption patterns and in the disparities between sexes regarding harmful drinking consequences. In the lack of statistical data targeting the reasons for the gender's disparity on alcohol consumption, we tried to identify some possible answers in scientific literature, as an important starting point for future researches. We think that these aspects are important because the control and decrease of alcohol consumption suppose a better knowing of cultural patterns that influence both men and women when they behave as prosocial or antisocial alcohol consumers.

\section{Gendered alcohol consumption patterns and potential explanations}

In order to create a larger perspective upon males and females patterns of alcohol consumption, we will start by presenting the results of a study which is interested on changes of gender drinking habits, over past decades. (Bratberget al., 2016). The authors start their analytical demarche revealing that, in traditional manner, males consume more alcohol and in more occasion than females (they also practice higher frequency of heavy drinking). In contrast with men, women are more likely to be lifelong abstainer and are less affected by harmful alcohol consequences. Disparities between the genders' alcohol consumption depend on cultural area but there are stronger influenced by men' alcohol consumption habits than that of women'. Relations among men' and women' alcohol consumption patterns are not clear and seem to be complicated. Probably, they are simultaneously due to biological particularities and to the specific gender roles culturally attributed within the society. The Authors shows that, in addition, other societal dimensions can influence gender gap: higher equality between sexes is associated with more similarity in alcohol consumption. Lowest disparities between sexes were identified in Nordic countries (including Norway) and the biggest ones, in states with developing economies. An interesting fact is revealed: age, school level, the work place, civil status and the childrearing are, each of them, aspects that influence the patterns disparities between men' and women' alcohol consumption but the specific effect is changing from one society to another.

The authors (Bratberg et al., 2016) highlight the fact that despite changes in gender roles and equality level between sexes which is manifested along with development of modern societies, gender disparities on alcohol consumption patterns remains important. Nevertheless, authors identifies facts that express the "convergence" of genders drinking patterns, convergence targeting the abatement of disparities on prevalence, frequency, or quantity of alcohol consumption. As an example, the gender drinking convergence, in USA, regarding quantities consumed, the frequency of binge drinking and rate of alcoholism was more 
obvious for young people than for the rest of the population. The convergence of gendered alcohol consumption habits found in Nordic countries seems to come from the rise of wine consumption, in an area where people typically drink beer and liquor (the introduction of a new beverage generates more acceptance for women that for men).It is revealed the fact that not in all cultures we can find alcohol consumption convergence between men and women. It is important to identify the way in which convergences found in different cultures depends on global changes of drinking consumption and to estimate what part of convergence is due to the change of drinking ratio between sexes.

The purpose of the field research (Bratberg et al., 2016) was to identify the evolution, in time, of men' and women' patterns of alcohol consumption using data from Norwegian "Nord-Trøndelag Health Study" (HUNT), which consisted in a longitudinal approach using successive cross-sectional surveys (in 1984-1986, 1995-1997, and 2006-2008), and using self-reported questionnaires. The conclusion of the researchers was that differences between sexes have been reduced for more aspects of alcohol consumption but the scale of this reduction depends on the age of the population. They analyze their outcomes in the context of the increasing access to alcohol for Norwegian citizen, due inclusively to the relaxation of alcohol control state policy. They believe, also, that the development of worldwide tourism makes alcohol cheaper and, also, that it influences cultural habits related to drinking: wine tends to replace liquors and, also, general consumption of alcohol tends to rise. A similar former study, named "GENESIS" reveals that Norwegian females and males have the same probability of being current drinker (in every age category) and between young people there are no differences regarding high frequency drinking. The authors highlight that between researchers targeting the evolution of alcohol consumption habits there are disputes about the fact that gender drinking convergence is determined by age, moment of the research or by cohort characteristics. It is concluded that always those three factors can be considered superposed. However, the effects due to birth could mirror historical impact and socialization process which influenced attitudes and behaviors lifelong practiced for people born at a specific moment of time. Authors' results indicate a significant decrease for most disparities between sexes in the majority of age groups in the last 20-30 years. They reveal, also, the presence of investigations which assumed that gendered alcohol disparities decrease if females' rights and their social status ameliorated by comparing to males' (Bratberg et al., 2016). They emphasize that there is a small number of studies on gender alcohol convergence centered on males' drinking but these offer some proofs of their consumption decline. As an example, the convergence was explained by reduction of alcohol consumption of men with high educational level. Another example comes from Germany and Switzerland and highlights that males reduced their hazardous drinking more than females between 1984 and 1992.The excessive attention on the increase in women' alcohol consumption was condemned because it was considered a gender stereotyping aiming to create a female negative image. Bratberg et al. (2016) emphasize that from data they analyzed result positive aspects for men, like the reduction with $50 \%$ of being drunk (within recent drinkers). It is also considered positive the fact that males change their preferences from liquors to beer and wine (which contain less alcohol).Women had, also, a benefic evolution 
on alcohol drinking within the category of 30-39 years (in which, most frequently, they give birth and take care of children), have a stable alcohol consumption, the share of abstainers rise and decreases drunkenness frequency. Problematic drinking acquired a higher incidence but the increase is lower than in the others age categories. Although there are some signs of temperance in alcohol using, for Norway people the share of persons having "lifetime problematic drinking" had increased for both sexes, mostly for women. Bratberg et al. (2016) highlight, on one hand, that the risk of getting alcohol problems or to become lifelong addicted is stable for males in numerous societies. On the other hand, scientific evidence from Sweden points that the alcohol problems (previously defined) increased for females between 1972 and 1997. Another significant fact (observed in Iceland) consists in the convergence of alcohol use disorders within mentally ill persons. Authors consider that this kind of transitions represents important reasons for concern, which contradict the optimistic conclusion of World Health Organization: decrease of social, medical and economic costs related to harmful alcohol consumption.

After a general approach targeting changes on gender alcohol disparities consumption we will try to identify some possible answers to the question: why do men and women have different patterns of drinking?

Lebreton (2016: 219 ) reveal the fact that high consumption of alcohol within USA and United Kingdom Universities was deeply analyzed, especially in relation with so-named "jock cultures". The same author offers a definition of "male jock culture" (Lebreton, 2016, p. 219, apud Macdonald \& Kirk , 1999: 132) as the "celebration of mesomorphy, anti-intellectualism, sexism, homophobia, competitiveness and binge drinking."'In concordance with the aspects just presented, the same author (Lebreton, 2016, p. 219 apud Skelton, 1993: 296) describes "male jock culture" as supposing "Getting drunk, fooling around, showing your naked body in public, displaying toughness, heterosexual conquest, respecting hierarchy and ridiculing weakness."

Sparks, Brown and Partington (2010) reveal that students at university level who study sport and physical education frequently define themselves and are defined by other people as "jocks" and participants to the "jock culture". This kind of culture was related to the generating and keeping of dominant forms of heterosexual masculinity through a set of social practices and ideological formations. Same authors noted that "jock culture" offers an "identity playground hierarchy" where the liberty to "play" with identities and to construct this identities is differently assigned depending on sex. They reveal the "12 Commandments" (Sparks, Brown and Partington, 2010, p. 334, apud Sparks, Brown and Brown, 2007) which drive, if there are respected, to a good integration as member of this type of culture: "1. Play high-level university sports; 2. Choose your sport wisely (as some sports afforded greater status of others; 3 . Only exceptional freshers (Year I students) make the first team; 4. Be committed to the social life; 5 . Excessive alcohol consumption and associated behaviors are obligatory; 6 . Respect the hierarchy; 7, Stay established; 8 . Look like a jock; 9 Attend social regularity; 10 . Attend post-match drinking session; 11. Credit for time served; 12. Gain position of power."

In order to reveal differences between male and female on alcohol consumption in a context of "Jock cultures", Lebreton (2016) realizes a survey report on French sport college students, which contain self-reported gender information. Secondly, the author collected qualitative data about the perception of both sexes on the use and misuse of 
alcohol in consensus with the frame of gender expressing. Analyzing data which came from the sample, Lebreton (2016) found that the proportion of males involved in formal sporting in is higher than females one's and that girls assign less time to sport training than boys. Regarding alcohol consumption, boys start earlier to drink and they have higher frequency of drinking, of alcohol intoxication and, in addition, their daily amount of alcohol consumption is superior to girls one's.

As we pointed, the author uses qualitative methods to reveal the way in which sports science students (boys and girls) speak about themselves as sportsmen and sportswomen, especially with respect to gender role structuring and alcohol drinking patterns. French students in sport science are characterized by a culture of heavy drinking, especially within males group (with a preference on beer). Masculine identification is often related to frequent beer consumption and, generally, to a high level of alcohol drinking. This could be the result of wish to generate a special "presentation of self” (Lebreton, 2016, apud Goffman 1990) in concordance with traditional "masculine" or "macho" sportsmen, who can drink excessive at parties, who can participate to all drinking games and remain capable to face the challenges of sports fields. In interviews with girls practicing sport were present, more frequent, topics like health, sexual hygiene, unpleasant reminding of being drunk and loosing of self-control, aspects which could justify females' rather moderate consumption of alcohol (compare to males'). Lebreton (2016, apud Peralta 2010) believes that girls' behavior it's different from boys' due to self-presentational aspects which are probably associated with stigma concerning women who are heavy drinkers.

An interesting perspective is offered by Foster et al. (2015) which analyzed Gender disparities on the incidence of alcohol use disorder for adolescents and Younger. Authors start their analytical approach by stressing that males consume more often alcohol and in bigger quantities that females and, as consequence, have more important incidence of alcohol use disorders (AUD). There are two kinds of gender effects - first related to average levels and, second, to structural aspects which support us to systemize our comprehension on relations between sex, AUD and the relevant psychosocial factors. Gender effects targeting average-level point out to the gravity of risk exposure encountered by male and female having AUD (by example, a risk factor may have a higher incidence for men than for women). Other significant source of differences between sexes is structural effects, respectively the intensity of the correlation among a risk factor and AUD separately by gender group. By example, gender disparities existing simultaneously for AUD and a psychosocial dimension can mask moderating effects of sex. So, a psychosocial dimension can be more strongly correlated with AUD in one gender group than in the other, regardless of the average differences between sexes for that dimension. In conclusion, risk factors on AUD with high structural effect can generate more powerful consequences for men or women and, therefore, it is needed less exposure to generate AUD. If it is controlled the level average effect of sex we can directly evaluate the intensity of the relation between every psychosocial dimensions and AUD. The delimitation of average-level and structural effects of sex belonging for diverse psychosocial dimensions can enlarge the comprehension of deterioration cumulating in different areas which may include pathways for AUD depending on sex. If it is controlled the average level effect of sex we can directly evaluate 
the intensity of the relation between every psychosocial dimensions and AUD. The delimitation of average-level and structural effects of sex belonging for diverse psychosocial dimensions can enlarge the comprehension of deterioration cumulating in different areas which may include pathways for AUD depending on sex.

Farther, the assessment of average level and structural effects at relevant moments for AUD evolution will be useful to detect models of psychosocial deterioration which concur to the offensive and persistence of AUD.

Foster et al. (2015) discuss, further, about typical risk factors of AUD and on their consequences. Authors highlight that AUD is the result of a lengthy process of interaction between biological, psychosocial and environmental risk factors, having reciprocal effects and being cumulative over time. The discussion is about variables which have gender differences at average level and, also, structural correlation with AUD. It is important the fact that this variables are characterized by gender disparities both at the average level and in the structural correlation with AUD. As example, authors indicate the uninhibited behavior involving innate personality characteristics of disinhibiting and, also, externalizing disorders. Such behavior patterns are generating higher rates of AUD' untimely beginning and, also, other conduct problems, like drug addiction, deviant behavior or early sexual activity. A succession of parent-child interactions characterized by using force or threats, disputes with socializing agents (such as professors or prosocial peers) and association with peers having antisocial behavior represent risk factors for AUD and difficulties associated with adulthood (lack of a workplace, couple relationship problems and low level of life satisfaction).Foster et al. (2015) reveal the existence of other risk factors associate in a non-specific manner with AUD, including internalizing disorders and experiencing traumatic life episodes, such as abuse of physical / sexual nature or person's attack. Although this risk factors and repercussions are usually correlated with AUD, there could be gender disparities regarding their average level and their intensity of the relation with AUD (representing structural effects).

Foster et al. (2015) discuss, further, about the gender disparities regarding the average level of risk factors for AUD. Studies point that men has higher risk on AUD than woman. To experience "Alcohol Use Disorder", women must have a more deviant behavior reported on their gender group standards compared to men. In these circumstances, the comprehension of gender disparities on risk and on effects for AUD demands a confrontation between the males and females having alcohol problems and the people of the same sex that do not have them. Authors reveal the fact that, frequently, risk factors are analyzed by comparing average level between men and women, fact that make heavier to find the specific role of those factors in the emergence of AUD both for males and females. As an example, authors reveal that despite the fact that average level of behavioral disinhibition and sexual trauma depends a lot on gender, these impairments have the same predictive power for AUD both in men and women. The comparisons realized separately by sexes between people with and without AUD can evaluate the relevance of a specific risk factor on AUD independently for male and for female. Authors highlight that this comparisons are essential to determine the predictive power of each risk factor in AUD for men reported to women. 
Foster et al. (2015) describe, further, the usage of gender disparities in analyzing AUD etiology. They reveal the small number of studies which compare the relative effects of various risk factors and their consequences for male and female, within the same research. If such tests are missing we can't decide if psychosocial risks and consequences are simply more frequent for one of sexes or there are some determinations of gender specific factors which make more intense their correlation with AUD within one gender group reported to the other. In order to evaluate the possible moderating role of gender, authors performed a direct confrontation between the effects of some risk factors and consequences of AUD within a important sample of male and female being between 17 and 29 years. More precisely, independently for each gender, it was initially evaluated the probability of having AUD at the age of 29, taking into account the average level of risk manifested for every risk factor at 17 years, after it was controlled the level of exhibit to risk factor separately for each of two sexes. Further, it was assessed the correlation from AUD and some psychosocial consequences at the age of 29, after it was adjusted gender disparities within basic rates of AUD and of psychosocial consequences. Researchers made the assumption that average level and not the structural effects of sex appurtenance will be identified at risk factors level.

As a conclusion, Foster et al. (2015) show that women with AUD have a higher risk at 17 years and that AUD grows average levels of negative psychosocial consequences for young adults, both male and female, but that internalizing distress is more powerful structural related with AUD for females by comparing to males. Higher level of exposure to every risk factor proved to be correlated with upper probability of having AUD at age of 29. In addition, male, generally, are characterized by bigger average levels of risk exposure which leads to upper prevalence of AUD for men by comparing to women. In spite of the bigger absolute risk for men, AUD of women has been correlated with a very important level of risk exposure for teenage period regarding their gender pattern and a superior level of risk exposure was needed for female to manifest AUD comparative to men. In addition, the disparities for deterioration level between AUD and nonAUD, at age of 29 (representing the effect size), were higher for females than for males. Compared with own gender pattern, female having AUD are inclined to live, on one hand, higher risk exposure in teenage period, and, on the other hand, consequences more unpleasant at the beginning of adulthood comparing to male with AUD. So, AUD seems to be a more serious mental disease in women than in men, with a risk pattern that starts earlier in girls' bio-social evolution. Authors identified some interactions between sex appurtenance and risk factors within teenage so that higher alcohol consumption, drugs use, deviant behavior in peer group and school difficulties augment the probability of having "Alcohol Use Disorder" much more for women than for men. Remarkably, the risks previously mentioned are not absolutely related with concomitant AUD presence, since the persons with AUD were identified through lifelong diagnoses at 29 years old. Authors conclude that AUD risk pattern seems to appear in teenage, regardless of the debut or chronic character of alcohol disorders. A more important AUD exposure risk for teenagers and repercussions related to AUD for young adult female offer supplementary arguments of the more severe and damaging nature of alcohol abuse for women comparing to men. 
Foster et al. (2015) consider, in concordance with others scientific researches, that the internalizing distress has a special structural connection with AUD for females (confronted with males) which indicates this aspect as a particular risk gender factor for AUD. For women, a higher internalizing distress will strongly augment the probability of having AUD in teenage and is meaningful associated with AUD in young adulthood. In contrary, for males, both teenagers and youth adult, AUD does not correlate with distress internalizing, which indicates that it is not a risk or an effect of AUD for men. Authors give an example regarding gender disparities on distress internalizing: depression and anxiety, psychological conditions more frequent at male than female teenagers, could be related with interaction problems at school, at job or within peer group (in pre-adolescence). Those conditions could catalyze drinking problems as a modality of facing unpleasant feelings. As a consequence, alcohol drinking could aggravate internalizing distress in an indirect way, by its bad effect on psychosocial evolution. Scientific literature reveals that is also possible that girls consume precocious alcohol and in important quantities without any relation with internalizing distress.

Alcohol misuse by teenager girls negatively affect neurocognitive functioning and could enhance isolation, ruin social interactions and may block school commitment. Instability of social support and low academic achievements can decrease girls' self esteem and their capacity of facing unpleasant feelings in a pro-social manner. Afterwards, internalizing symptoms can appear when teenagers became young adults. For clarifying the dimension of women vulnerability at AUD it will be need to directly evaluate the temporal association among internalizing distress and drinking problems by using a longitudinal analysis. As a final conclusion Foster et al. (2015) state that, at general level, they sustained scientifically that AUD is a worse disease in women and, also, that internalizing distress is possible to have a specific sex role in AUD medical signs within females group.

Evans et al (2017) examine another dimension of gender disparities, targeting the relation between childhood adversity and substance use disorders (with possible moderator effect of gender). Authors reveal that substance use disorders (SUD) have one of the highest prevalence from USA and men have a bigger probability that women to consume excessively or became addicted on alcohol or other drugs (10.7\% comparative to $5.7 \%$ ). Gender disparities were found in previous aspects, associate dimensions and consequences of SUD. Authors highlight that some researches indicate that women, comparing to men, use more frequent illegal drugs to face unpleasant emotion, traumatic life events, including childhood abuse (of physical or sexual nature).

The term of „Childhood adversity” is described as abuse, slighting and familial dysfunction manifesting under the age of 18 . Studies from this domain show that childhood adversity is associated with a number of health problems, including SUD. In USA, substance abuse of parents had the highest frequency within the types of ,childhood adversity" and, in addition, children of parents which consumed drugs had a higher probability of SUD in teenage and in adult period.

Evans et al (2017) reveal that, relative to drugs, alcohol is more accessible, less socially stigmatized and considered as less dangerous. The majority of studies show that men consume alcohol more frequently and in bigger quantities than women. By opposition, the differences between sexes regarding SUD are small in 
the general population, fact due to the small gender disparities in the proportion of stimulants use and in the misuse of sedatives and opioides. Simultaneously, women, relatively to men, are confronting with a rougher social stigma, which is connected more intense with drug use than to alcohol abuse. In consequence, regarding the background of childhood adversity, male and female can, probably, face comparable risk for AUD but it is likely that woman encounter bigger risk for SUD. Authors highlight that previous studies on this domain were not able to reveal the specific gender disparities regarding the consequences of childhood adversity, which might be used to adapt AUD prophylaxis and intervention efforts.

The aim of the study (Evans et al, 2017) was to analyze gender disparities regarding the correlation among childhood adversity and different kinds of SUD and, also, to find if gender moderates this connections. The research method supposed analyzing of data coming from the second part (2004-2005) of the National Epidemiologic Survey on Alcohol and Related Conditions (NESARC). It was used multinomial logistic regression, weighted to be statistically representative for American adults and it was calculated predicted probabilities by gender, controlling for covariates.

As primary results, authors highlight that over $50 \%$ of American males and females were exposed to childhood adversity. In addition, there were more women than men exposed to at least 3 kinds of childhood adversity and a higher proportion of women confronted sexual abuse in childhood. Evans et al (2017) revealed that, for each kind of SUD, females had a less risk than male and, also, that it is a strong relation between the exposure to childhood adversity and the behavioral response of both sexes. More different kinds of exposure to childhood adversity decreases gender disparities regarding the risk of having alcohol use disorder. If people were exposed to many kinds of childhood adversity, the differences between genders decrease regarding to drugs; for people who lived 3 or more different kinds of childhood adversity, the probability of having SUD is bigger for women than for men. In consequence, the increase of the number of kinds of childhood adversity at which people are exposed leads to equalization of women' and men' likelihood of having SUD or even to the situation in which females exceed males.

In reverse, experiencing of more kinds of childhood adversity reduce the gender disparity in probabilities of having AUD and enlarge the gender gap on SUD risk. As an explanatory model, authors show that childhood adversity makes women more vulnerable than men to the consequences of adult stressors. As an example, they indicate the deterioration of stress-response coping mechanisms and decrease emotional regulation, which augment adult females risk to SUD. As a consequence, in the lack of childhood adversity, the reactivity to stressors was not influenced, so the probability of SUD emergence is lower for women, in concordance with cultural norms targeting gender roles expectations and occasions to use drugs. The fact that the exposure at more kinds of childhood adversity was related to higher SUD risk especially for men was explained through the disparity between the motives which determine young male and female to start drug use. Authors assume that childhood adversity may determine negative self-emotions (blame, disgrace, self-devaluation) which are frequently externalized, by boys, as aggressive and violent behaviors and who, in consequence, use drugs to defy the society. In opposition, the childhood adversity exposure is more frequently 
interiorized as anxiety, depression, and social retraction within girl groups, who use drugs as self-medication. Evans et al (2017) consider that evidence they found suggest that the decreasing of different kinds of SUD, both for men and women, necessarily implies prevention of childhood adversity. In addition, would be necessary gender-specific interventions in order to support people to face these types of adversity when it appears. There are necessary some changes in health care policy in order to identify and treat a greater number of persons at risk for SUD within primary care institutions.

Jalling et al. (2017) approach gender disparities on alcohol misuse from another point of view. They highlight the important correlation between teenager's dangerous alcohol consumption and their perception on peer' conduct, their personal way of using money, respectively several kinds of deviant behavior. Authors observe, in addition, the poor number of studies that analyze gender-specific predictors within teenagers' deviant groups (including alcohol users) in order to design an efficient gender selective intervention. The purpose of the research was to evaluate shortterm predictors of Heavy Episodic Drinking (HED) and hazardous use of alcohol for teenagers (12-18 years) of both sexes with delinquent conduct and which are alcohol users. The population of the research consisted in 85 girls and 77 boys, together with their parents, evaluated at the beginning of study and after 6 month with some standardized psychological tools aiming to measure externalizing and internalizing conduct, alcohol drinking, psychological and social distress and the deviant behavior. More precisely, the authors wanted to achieve the following goals: 1 . to assess deviant conduct and psychosocial dysfunctions as predictors for HED and the risky alcohol use; 2. to check if parental evaluation of teenagers' deviant conduct represent a predictor for HED and the risky alcohol use; 3 . To analyze if the teenagers' way of spending money, perceived parental wealth, deviant conduct and alcohol use of peers, sex and/or age are predictors for HED and the risky alcohol use.

First results (Jalling et al, 2017) show that, contrary to previous researches, the perception of peer alcohol consumption has the same effect on HED and risky alcohol use for both sexes. Most important results show that, for vulnerable teenagers, perception on peer's alcohol consumption is important for the incidence of hazardous drinking at both sexes. In addition, females' risky alcohol use was adequate predicted by their externalizing conduct.

Moreover, norm violation and teenagers' social difficulties are predicting both Heavy Episodic Drinking (HED) and risky alcohol use for men. In women case, norm violation predicts only risky alcohol use while social difficulties predict only HED. In the same vein, family evaluation of teenagers externalizing conduct predicts only risky alcohol use of their children. Data collected by researchers (Jalling et al, 2017) indicate only a few behavioral problems as predictors for HED and for risky alcohol use but, more important, prediction pattern is similar both for male and female. Research results validate the idea that both boys' and girls' perception upon peer alcohol consumption represents a predictor for the future alcohol use to the same extent for male and female. In contrast to other studies, current research did not find teenagers' age as a predictor for HED or risky alcohol use.

Zimmermann et al. (2011) approach gender alcohol use disparities by analyzing individual differences in instrumental and expressive traits (gender-stereotyped) 
which represent the possible influence of male and female gender role. This influence could explain a part of variance characteristic to male alcohol use. Authors' research refers to the relation between gender aspects and alcohol consumption of males from Germany and Spain; data were collected transversal, using questionnaires which targeted gender-related trait. It is important to take into account that gender role variables could explain a significant part of the variance for alcohol use between male and female and within each gender group (between its members). So, each person is possible to be different from another regarding alcohol drinking according to their consonance with the socio-cultural definition of the two genders. To express their masculinity, men had to accede at behaviors cultural defined as masculine and refuse what is defined as feminine. Consumption of alcohol is related to characteristics of male role as risk taking, dominance, resistance, and invincibility. However, certain researchers found moderate positive correlation between masculine traits (in Conformity to Masculine Norms Inventory) and health-risk behaviors and also, a moderate negative association with healthprotective behaviors. As a result of scientific literature reviewing, authors highlight the existence of more and more facts that sustain the correlation between gender roles and alcohol use. Zimmermann et al. (2011) consider that is important to approach a large cultural context when are analyzed gender aspects because it is probable that societies differ in great extent regarding the support for traditional gender norms. Differences occur, also, in social structuring gender norms regarding health-behavior. Authors reveal that taking into account of culture can drive to not expect outcome. There is a scientific study which reveals that instrumental features were related to lower quantities of alcohol use by Russian males and that expressive traits were associate with women' higher consumption of alcohol in Canada. Authors highlight the importance of the cultural background and the attention to be paid to the results generalization validity. In their field research, Zimmermann et al. (2011) pursued to enlarge the former studies regarding the relation between gender roles and alcohol drinking of men (from Germany and Spain). Authors found that daytime alcohol consumption was more frequent in Spain comparing to Germany but they did not identify differences regarding evening consumption. More important, they did not found disparities between Spanish and German sample with respect to the average of gender-related personality traits. The consumption of night-time alcohol was predicted, however, by instrumental and expressive features, activity which can be interpreted as being "masculine" as long as predictors from each countries concord with the expectations existing in traditional gender-role stereotypes. Consumption of a few drinks in the first part of day was more usual in Spanish male which had more intense instrumental traits, and less frequent for German men having higher level of expressive features. Authors result, referring to Spanish male, are in consensus with scientific approach of gender roles and men's health habits and assume that males who interiorize traditionally masculine traits become practitioners of stereotypically masculine behaviors, as alcohol drinking. An important finding in Zimmermann (2011) study is the protective role of the expressiveness, which is correlated with lower quantity of alcohol consumed daytime (for both studied nationalities) and with more rare alcohol drinking by nighttime for German male. The authors assumed that males which have a higher expressiveness are more responsive to their body and to their somatic marks in comparison with one having small level of expressiveness and, 
therefore, they choose to restrain their consumption to a limit perceived as natural (which is not dangerous). An interesting observation is the fact that ,androgynous people", having high level of instrumentality and expressiveness traits, consume smaller quantities of alcohol and tobacco than males with high instrumental features but low expressive characteristic. Zimmermann et al (2011) assume that men characterized by high level of expressiveness reduce their alcohol consumption in order to diminish unpleasant feelings of other people which could see them implied in heavy drinking episodes. Author reveals the importance of gender role evolution, in concordance with social change of cultural norms, such as the use of paternal leave, determines men to behave in a less gender-stereotypical way. So, men behavior regarding alcohol consumption would be at the intersection of internal characteristics and the social prescriptions targeting gender role. Scientists from different domains share a constructivist perspective on gender which derives from: 1 . the distribution of social roles; 2 . the practice of health-related behaviors; 3. consumption of alcohol as a masculine act. The relations existing on one hand, between alcohol consumption and masculine traits (strong personality, inclination to take risks) and, on the other hand, between low frequency of drinking and the feminine characteristics (tenderness, compassionate), relation which were observed both on German and Spanish men, are in consensus with the universal character of gender stereotypes.

\section{Analysis purpose}

As a general purpose, in this study we intend to evaluate the gender gap concerning alcohol consumption and to reveal men and women disparities regarding harmful drinking consequences from a comparative European view.

The specific objectives that we proposed were the following:

- To evaluate gender gap regarding alcohol consumption (quantity, frequency, binge drinking)

- To compare, in present, Romanian gender gap in alcohol consumption with other countries from EU

- To find some of most harmful effects of alcohol consumption and to present, by that perspective, men and women situation, in Romania and in EU

- To identify relations between global alcohol consumption and the amount of gender gap regarding pattern of consumption and harmful effects of drinking

Of course, we could formulate objectives related to the evolution, in time, of aspects pointed before but we consider that this would be another subject of research, making reference to much more complex realities such as, for example, the general evolution of stereotypes targeting gender roles. 


\section{Methodology}

The method used in our research was the secondary analyze of statistic documents which were provided by EUROSTAT Database and by World Health Organization (WHO) through its structure, Global Information System on Alcohol and Health (GISAH). We accessed, also, data from a report of WHO, named „Global status report on alcohol and health 2018" from which we selected the most important information about alcohol consumption of men and women in 2016 at global level.

\section{Results}

In the first place I would like to present alcohol consumption in European countries, in 2016, per total and separately by gender in order to reveal Romanian place. From figure nr.1 we can see that alcohol consumption, in our country, is one of the highest in Europe, occupying the $8^{\text {th }}$ place (in descending order of total drinking).

We can see, in addition, that, in each country, men drink more than women but the gender gap decrease when both sexes consumption rises.

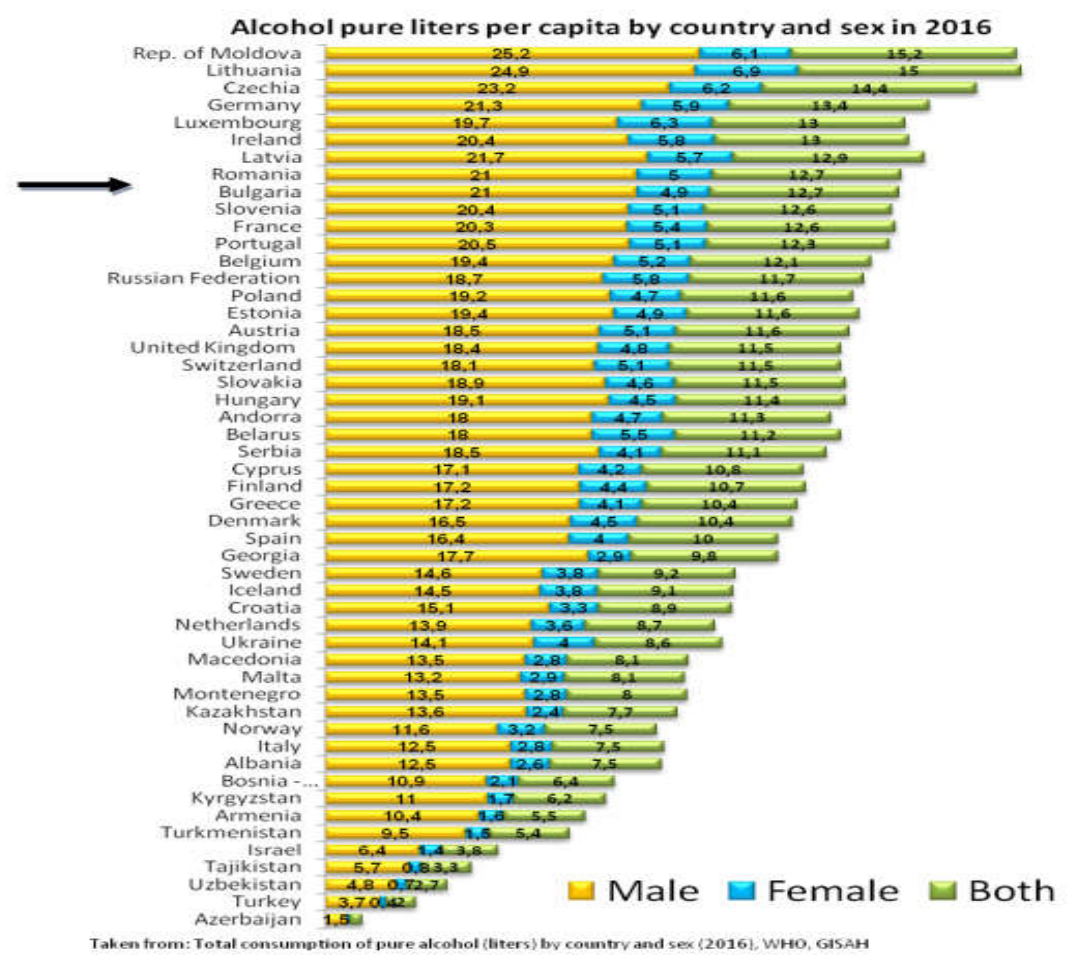

Figure 1. Total consumption of pure alcohol (liters) by country and sex (2016) 
There is a strong negative correlation $(r=-0,804)$ between total alcohol consumption and the number indicating how many times men drink more than women. The next aspect regarding gendered pattern of alcohol consumption consist in the frequency of the behavior. This data come from Eurostat and cover only 2014 year.

\section{Frequency of "DAllY" alcohol consumption by sex, 2014}

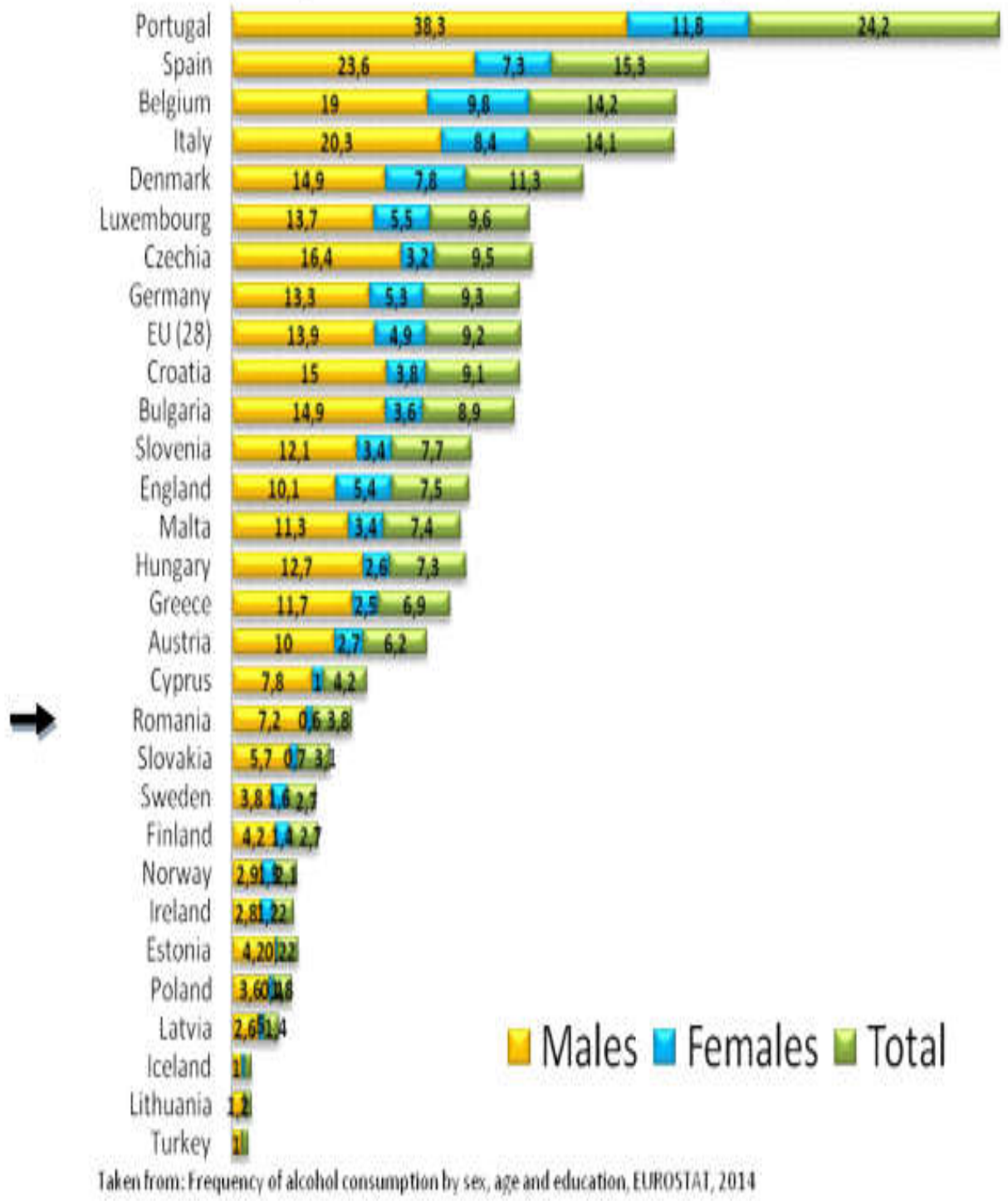

Figure 2. Frequency of alcohol consumption by sex (DAILY) in 2014 
We can see that Romania, at that time, occupies a good position, $19^{\text {th }}$ place (in descending order of percentages) from 30 countries (EU+2, in 2014). It is interesting, also that there is an important negative correlation $(-0.435)$ between the percent of people who declare that they consume alcohol daily and the number that indicates how many times men do this thing more often than women. Romania position became even better if we consider the cumulate proportion of people who declared that they consume alcohol „daily” or „,weekly”. In this case, Romania stands on place 26 of 30 countries (by descending frequency of mentioned cumulate percents).

We can see, already, that, beside the large gender gap between men and women on alcohol consumption in Romania, a pattern of consumption emerges: when the quantity or frequency of alcohol consumption increases, the gender gap tends decrease. In order to clarify alcohol consumption patterns in Romania, we present the proportion of people which drink very low quantities (who didn't consume last month or last year or which are abstinent) for the entire population and by genders.

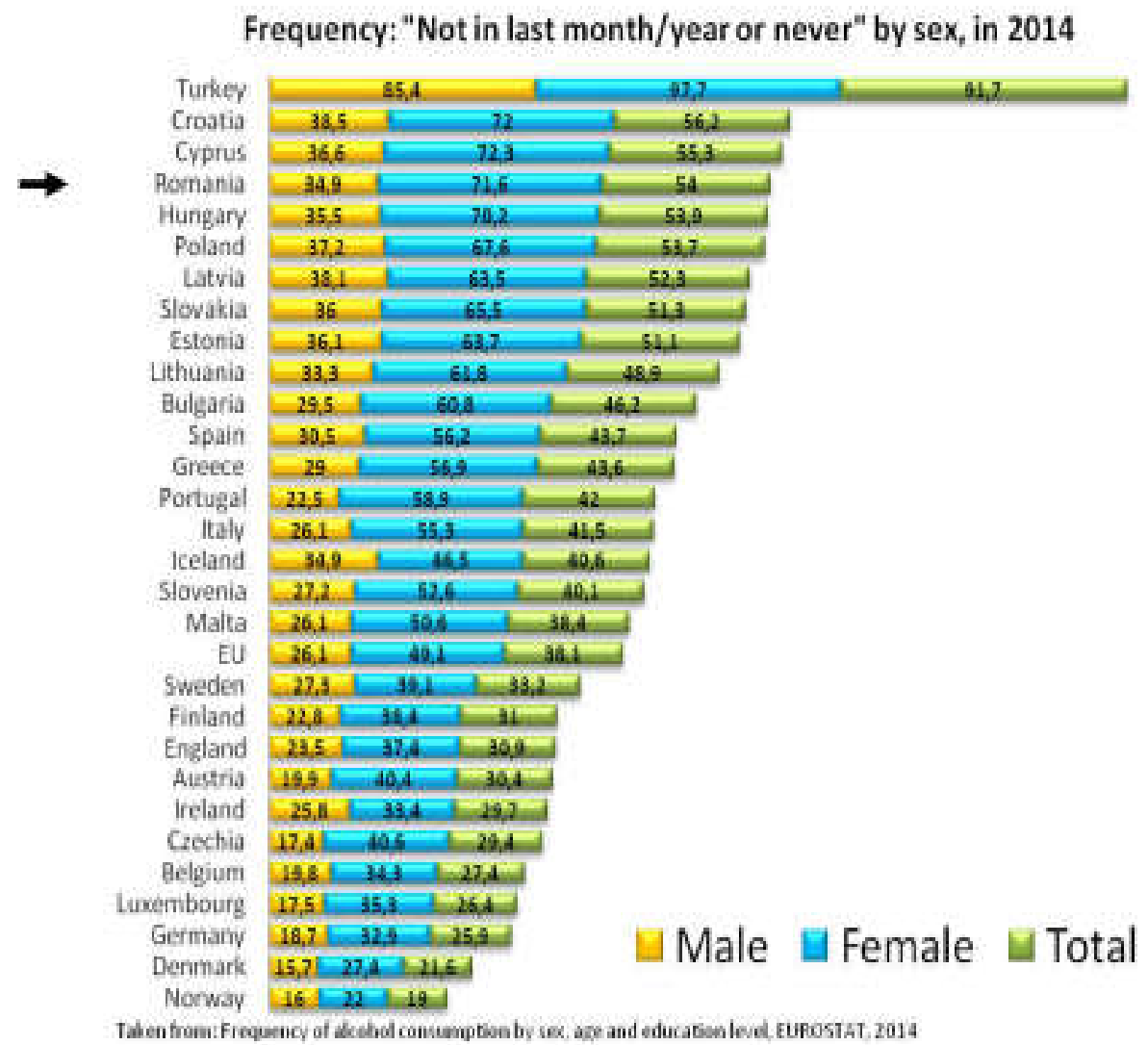

Figure 3. Frequency of alcohol drinking by sex (Not this month/Year or Never) in 2014 
In Figure 3 we can see that Romania occupies the $4^{\text {th }}$ position, between 30 European countries, with respect to the cumulate proportion of people who declared they didn't consumed alcohol last month, last year or never. In Romania low level alcohol consumers are twice more frequent between women comparing to men. At European level we can see that as it grows the proportion of low alcohol consumers, as the proportion of males divided to females tend to decrease (but the relation is moderate, $r=-0.221$, and for a sample of 30 elements would not be statistically significant).

The data presented in figure 3 must not be confounded with those regarding Romanian position in Europe on the proportion of lifetime abstainers. In concordance with data provided by WHO on year 2016 situation, Romania lies on $25^{\text {th }}$ place (from 51 countries) according to the total percent of abstainers. The correlation coefficient between the proportion of total abstainers and the rapport between men' and woman' abstainers is $r=-0.73$ which show that, generally, gender gap regarding the share of abstainers decrease as it grows the proportion of people who never drink.

Another important aspect of human behavior regarding alcohol are the ,heavy episodic drinking", which may be, partly, responsible for the incidence of some alcohol harm (especially injury, violent episodes, etc.).

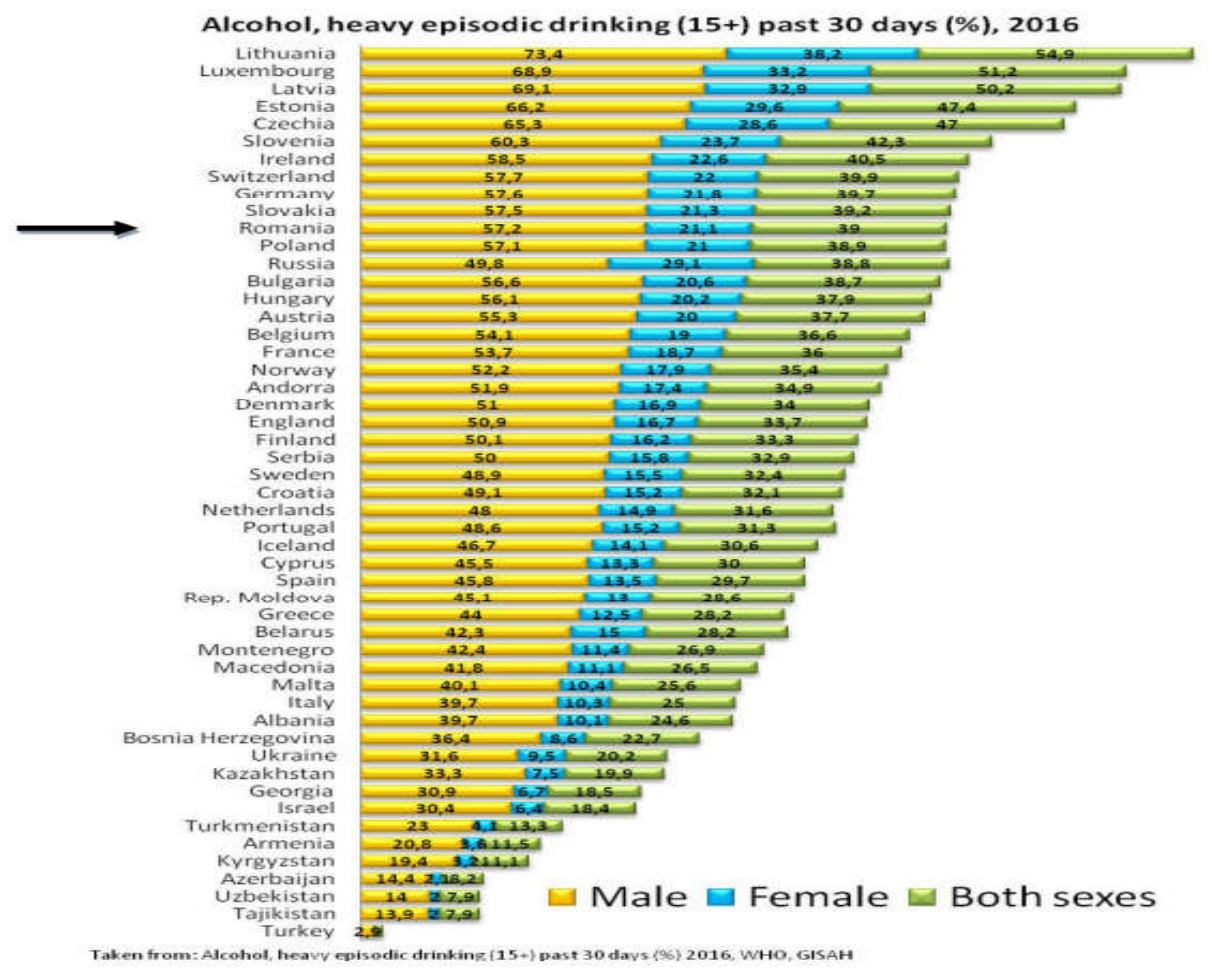

Figure 4. Alcohol, heavy episodic drinking (15+) past 30 days (\%) (2016) 
We can see, at this time, that Romania is one of the countries with highest prevalence of ,heavy episodic drinking”, fact that may explain the discrepancy that we observed between alcohol consumed per capita and the low frequency of drinking, respectively the high proportion of lifetime abstinent. There is, probably, a relatively small group of persons who are drinking big quantities of alcohol during a year and who, also, drink large quantities at one occasion. It is interesting that these persons are not necessarily alcoholics, because the proportion of person addicted to alcohol is not big in Romania. We are talking, simply, about men and women who consume alcohol excessively, with high frequency and in large amount but not daily.

It is important to note that, like in others aspects describing gender differences regarding pattern of alcohol consumption, men have a much more risky behaviors. With respect for „heavy episodic drinking”, $57.2 \%$ of male declared minimum one behavior of that nature last 30 days while the percent of female was only $21.1 \%$. This observation must be completed with the fact that the total proportion of „heavy episodic drinkers" correlates with the number indicating how many times men, compared with women, have more frequent the previous mentioned behavior (the correlation coefficient $r=-0,822$ ).

Last aspect that we will present regarding the incidence of alcohol misuse is the frequency of ,heavy episodic drinking” for younger (15-19 years) which represent, obvious, a special group of people who present a particular interest because most alcohol problems start in adolescence and become more and more serious as time pass away (most eloquent example: people became addicted).

For the population having 15 years or more, Romania position is the $11^{\text {th }}$ from 51 countries in what concerns the frequency of ,heavy episodic drinking”. For young people the similar position is the $13^{\text {th }}$ (the prevalence is $52.9 \%$ for boys and $17.1 \%$ for girls, only a little lower than for the entire population - males $57.2 \%$, females $21.1 \%$ ).

Analyzing figure 5 we can find, again, that masculine population has a much more risky behavior regarding alcohol consumption (remark valid with respect to „heavy episodic drinking" but, also, for the other patterns of alcohol consumption that we discussed before). We can see, also, that the disparities between genders decrease as alcohol misuse frequency became more important. The coefficient of correlation between the proportion of younger that declare minimum one "heavy episodic drinking" past 30 days and number showing how many times a male practices that behavior more than a female is $r=-0.810$, closely to the value that was obtained for the entire population (having 15 years or more).

We are highlighting, at this moment, the idea that if a relatively large share of population would practice a certain behavior relative to alcohol use, the difference between men and woman would be low. On the contrary, if a pattern of alcohol consumption is practiced by a relatively small proportion of the population, it is very probably that, within that group, men are many more than women. We must add that this observation is valid both for positive perceived and for negative alcohol related conducts. For example, the correlation coefficient between the proportion of total abstainers and the rapport between men' and woman' abstain is $r=-0.73$ which show that, generally, gender gap regarding the share of abstainers decrease at the same time with the increase of the proportion of people who do not consumed alcohol their entire life. 


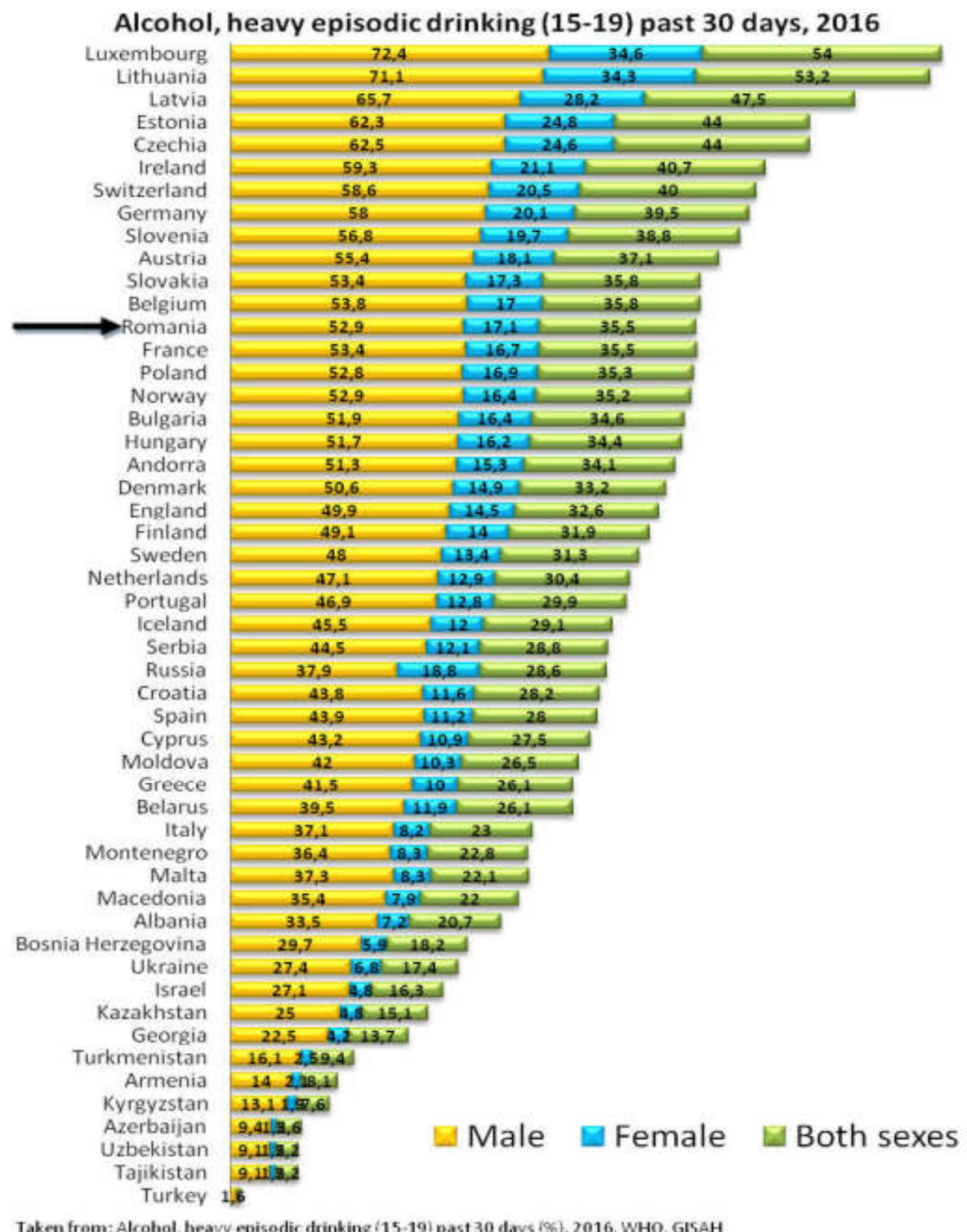

Figure 5. Alcohol, heavy episodic drinking (15-19) past 30 days (\%)

From this moment we will present the harmful consequences of alcohol consumption for the entire population and separately, by gender. 


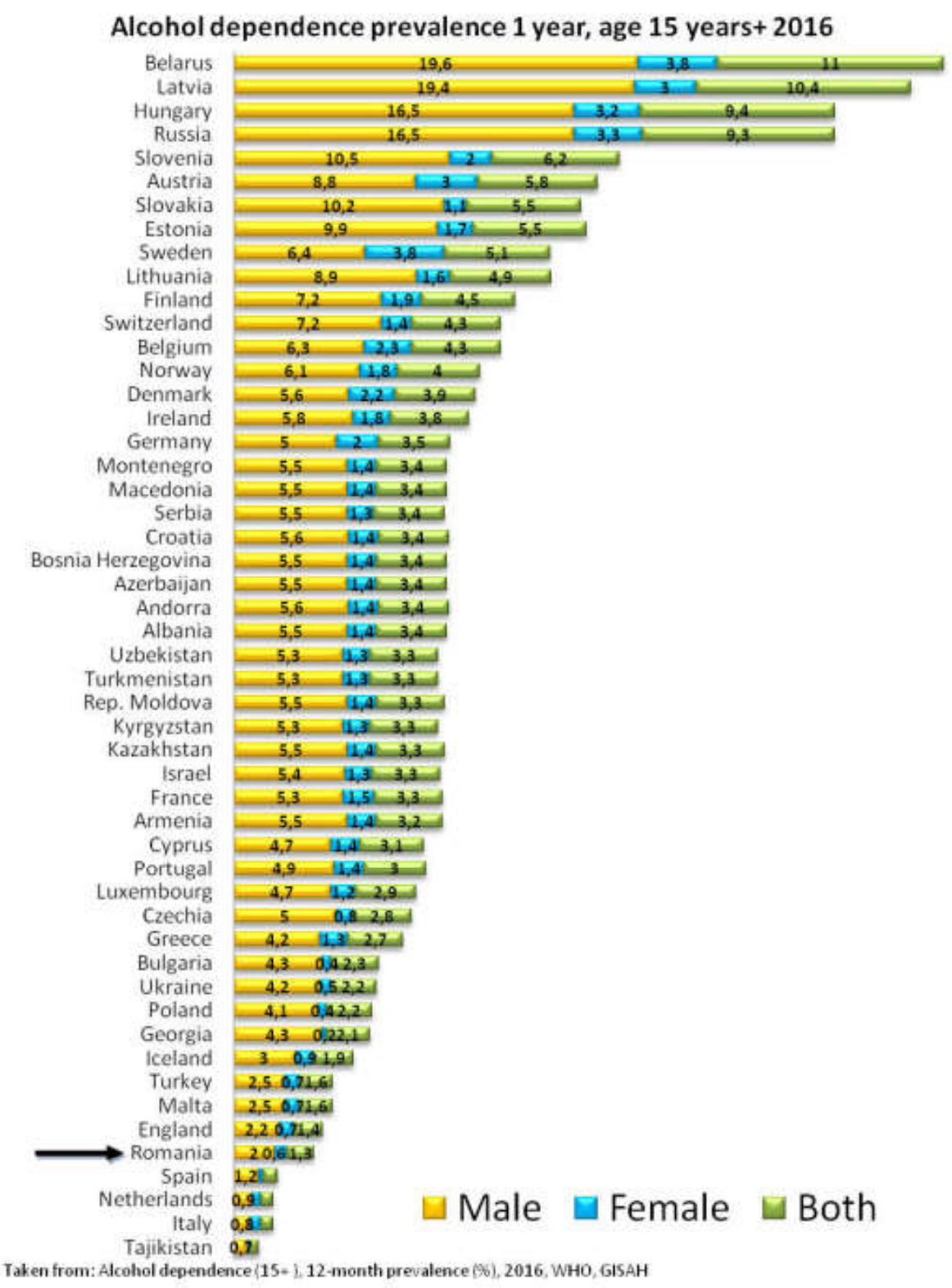

Figure 6. Alcohol dependence (15+), 12 month prevalence (\%)

Regarding alcohol dependence Romania occupies a very good position, 47 of 51 countries ranked in descending order of alcoholism prevalence. In Romania the proportion of alcoholic males is 3.3 times higher that of alcoholic females. At EU level the correlation between the global proportion of alcoholism and the 


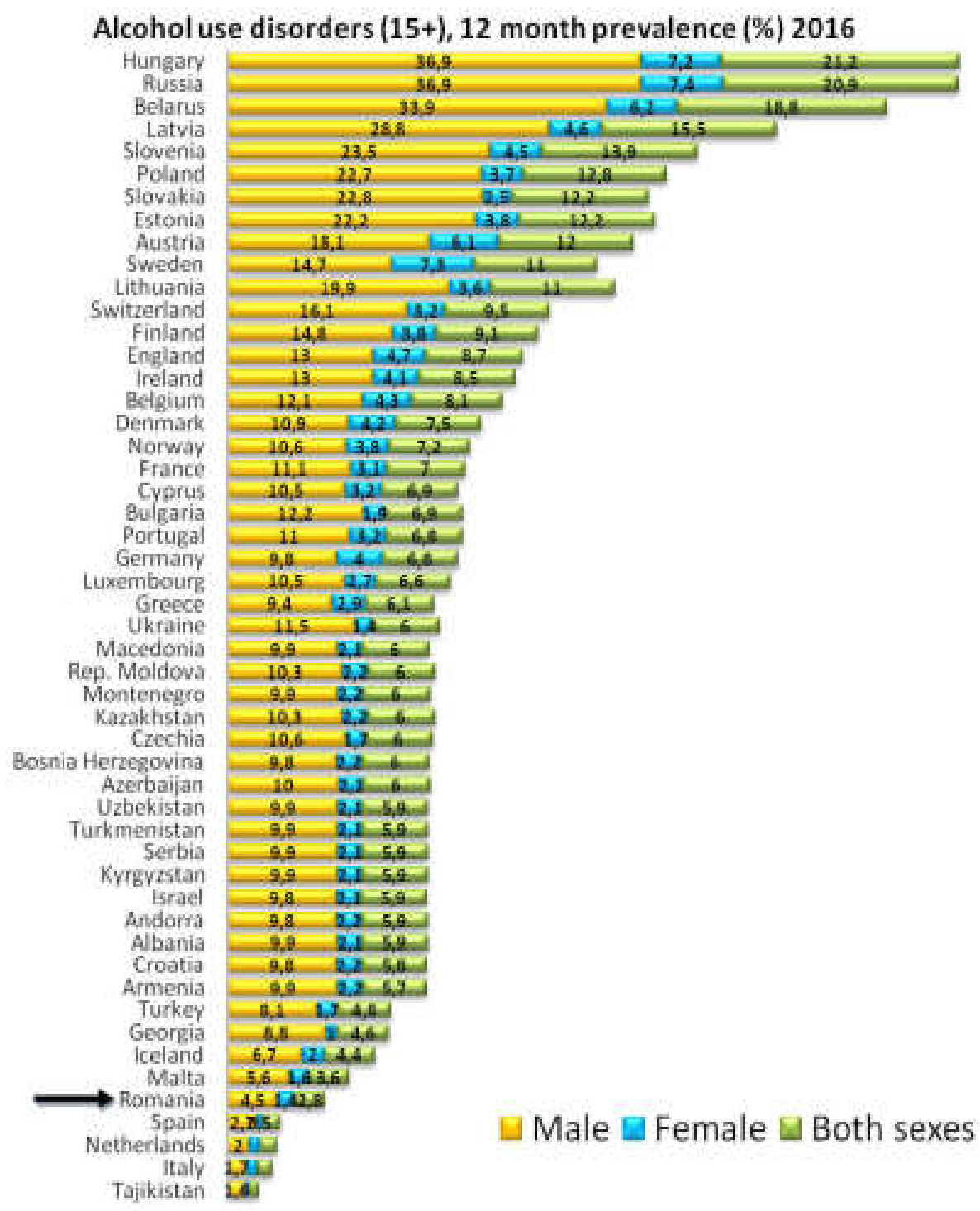

Taken from: Alcobol me disordes $(15+1,12$ monthprevalence /8), 2016, WHO, GISAH

Figure 7. Alcohol use disorders (15+), 12 month prevalence (\%)

With respect to alcohol use disorder (AUD), Romania occupy position 47 of 51 countries ranked in decreasing order of person proportion having AUD. In Romania the prevalence of AUD is 2 times bigger for men by comparing to 
women. In addition, we found a positive correlation $(\mathrm{r}=0.227)$ between the share of people with AUD and the ratio of proportion characteristic to men respectively, to women. Regarding alcohol consequences, increasing prevalence seems to increase gender disparities.

\section{Alcohol attributible fractions, all-cause deaths (\%), 2016}

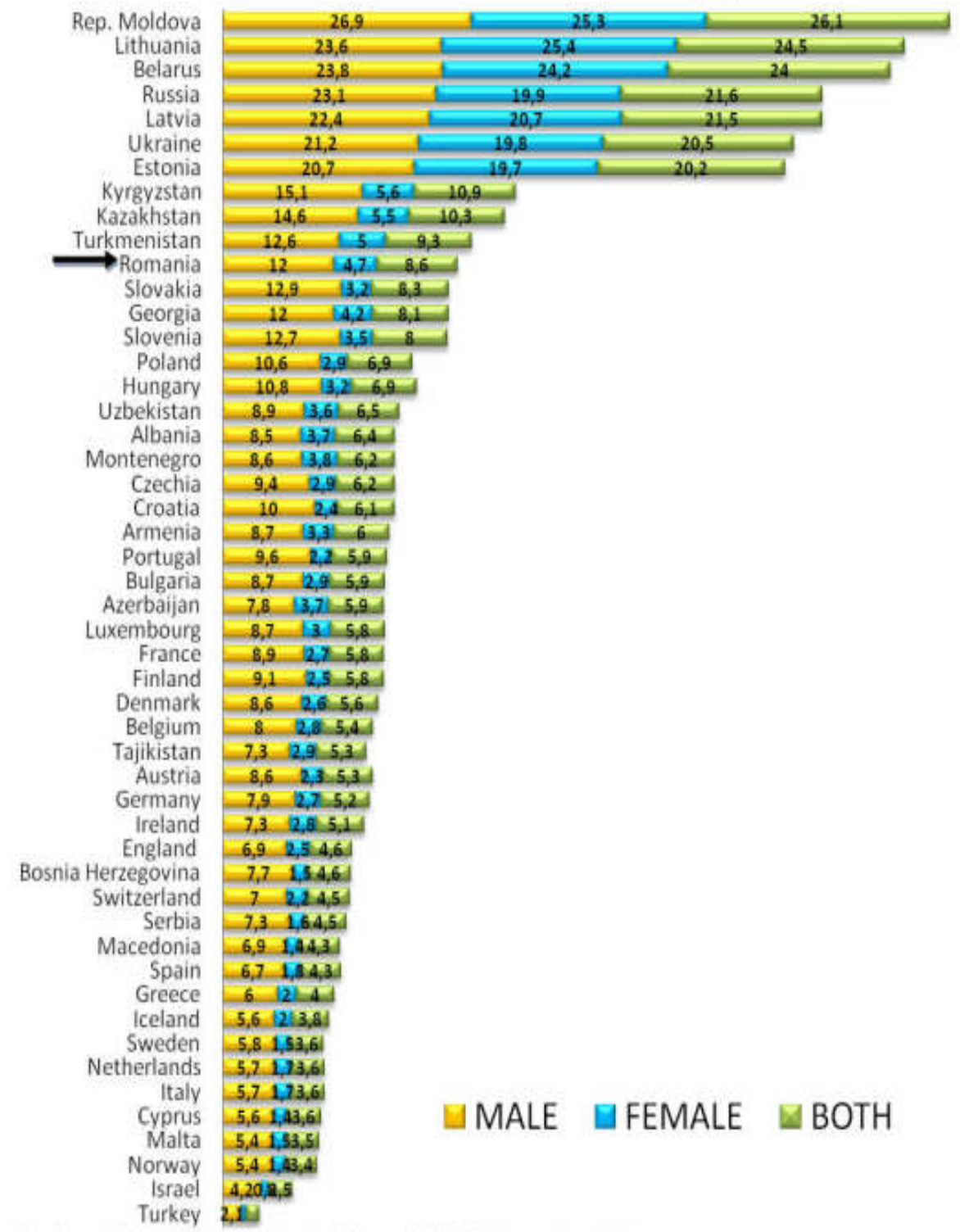

Taken from Alcohol-attributable fractions, all-cause deaths $\{\%\}, 2016$, WHO, GISAH

Figure 8. Alcohol-attributable fractions, all-cause deaths (\%) 
Concerning the fraction of deaths attributable to alcohol Romania has a relatively bad place ( 11 of 50 countries ranked in descending order of the criterion that we mentioned before). The correlation between the proportion that express ,Alcoholattributable fractions, all-cause deaths" and the rapport between the same aspects characterizing men, respectively women is $r=-0.794$.

\begin{tabular}{|c|c|c|c|}
\hline \multicolumn{2}{|c|}{$\begin{array}{l}\text { Alcohol-attributable fractions } \\
\qquad(15+) \text {, cancer deaths }(\%)\end{array}$} & \multicolumn{2}{|c|}{$\begin{array}{c}\text { Alcohol-attributable fractions (15 } \\
\text { liver cirrhosis deaths }(\%)\end{array}$} \\
\hline Rep. Moldova & 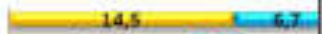 & Crechia & so,2 \\
\hline Slovakia & $21,5, \quad[a, 3 \mid$ & Bulgaria & $39,2 \quad \mathrm{H}=62,1$ \\
\hline$\Rightarrow$ Romania & 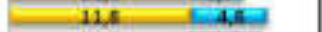 & fromania & $78,9 \quad 62,1=$ \\
\hline Ukraine & $15,6 \quad-4,6=$ & Portugal & 61 \\
\hline Hungary & $-11,4 \quad \mid 1,40$ & Germany & $74,6=66,4$ \\
\hline Belarus. & 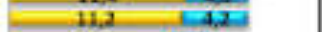 & Ireland & 74.2 la $\quad 62=$ \\
\hline Turkmenistan & $10,5 \quad$ a. & Slovenia & 24,2 a $12.5=$ \\
\hline Kazakhstan & $\begin{array}{rr}-20,1 & 1,4\end{array}$ & Poland & $78,1 \quad 62,2$ \\
\hline Russia & $10,1 \quad-4=5,5$ & France & $77.9 \quad-66,4$ \\
\hline Portugal & $9,2 \quad-4,2 a$ & Slovakia & $77.5 \quad 62$ \\
\hline Lithuania & $9.7 \quad 15.47$ & Hungary & $77,5 \quad$ in $\quad 60,7=$ \\
\hline Latvia & $9,3 \quad 1,3,29$ & Georgia & 72,4 In $\quad 47,1$ \\
\hline Croatia & $9,2 \quad \mid 3,0:$ & Serbia & $-27,3 \quad f-52,4$ \\
\hline Kyrgyzstan & $\begin{array}{ll}2.4 & \text { M.5. }\end{array}$ & Belgium & $\begin{array}{rr}27,1 & 63,9 \\
\end{array}$ \\
\hline Crechia & $0,1 \quad=0,0$ & England & 72 in $-62,4$ \\
\hline Bulgaria & $0,1 \quad$ 1.1.4. & Luxembourg & $364 \quad \mathrm{~m}=\mathrm{sa}$ \\
\hline Serbia & $3,5 \quad B, 3,4$ & Austria & 353 in 63 \\
\hline Ireland & 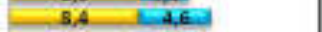 & Finland & $\begin{array}{r}75,7 \\
-61,1\end{array}$ \\
\hline Spain & a,9 $\quad 1,9,9$ & Switzerland & $74,9 \quad 53,3$ \\
\hline Slovenia & a,3 $\quad \mid, 3,3$ & Greece & $749-561$ \\
\hline Poland & $\begin{array}{ll}1,2 & 12,93\end{array}$ & Crprus & $74,2 \quad 1=57.8$ \\
\hline Austria & $\begin{array}{ll}1,2 & \text { M,fit }\end{array}$ & Denmark & $74,3 \quad$ - $\quad=0,3$ \\
\hline France & $5,1 \quad$ b. $4,0.0$ & Kazakhstan & $73,9-1-45,2$ \\
\hline Germany & $5.0 \quad 4.00$ & Spain & $73,5 \quad 56,1$ \\
\hline England & s.0 latila & Croatia & $\begin{array}{ll}73,4 & 52,5 \\
\end{array}$ \\
\hline Uztsekistan & $7,5=-4,3$ & keland & 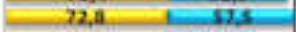 \\
\hline Estonia. & 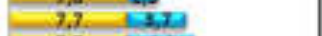 & Kyrgyzstan & 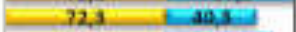 \\
\hline Bclgium & $2,7 \quad$ l. $4,2$. & Montenegro & 71, \\
\hline Denmark & $2.5 \quad-2.5 .5$ & Sweden & 703 in ind \\
\hline Montenegro & $2,1 \quad 6,2,1$ & Albania & $70.3 \quad-45.6$ \\
\hline Switzerland & $2 \quad+3,23$ & Netherlands & $69.7 \quad$ in $\quad 555$ \\
\hline Bosnia & $\begin{array}{ll}6,2 & 12,2\end{array}$ & Malta & $69.2 \quad 1=50.8$ \\
\hline Netherlands. & $6,8 \quad 53,14$ & Armenia & 69,2 In 34,7 \\
\hline Luxembourg & 6.2 \&.6.1. & Turkmenistan & \begin{tabular}{r|r|}
69 & $30,5=$
\end{tabular} \\
\hline Tajikistan & $6.6 \quad 1.5$ & Bósnia & $65,6 \quad$ In $43=4$ \\
\hline Macedonia & $\begin{array}{ll}6.5 & 12.3\end{array}$ & Italy & $67,6 \quad$ In 48,7 \\
\hline Georpia & $\begin{array}{rr}6,4 & 12,2\end{array}$ & Norway & 63.4 ol 54.7 \\
\hline Finland & $6,3 \quad 43,6$ & Tajikistan & 60,5 fas, \\
\hline Malta & $6,2 \quad 12,4$ & Uzbekistan & $\begin{array}{ll}58,6 & -29,21 \\
\end{array}$ \\
\hline itahy & $\begin{array}{ll}5,1 & 12,5\end{array}$ & Rep. Moldowa & $52 \quad$ the 625 \\
\hline Sweden & $\begin{array}{ll}6 & 023\end{array}$ & Lithuania & $53.6 \quad 62.6$ \\
\hline iceland & $\begin{array}{ll}4,5 & 12,7\end{array}$ & Israel & $528=152$ \\
\hline Greece & $\begin{array}{rr}-5,5 & -1,5,5\end{array}$ & Belarus & $52-1=63.6$ \\
\hline Norway & $\begin{array}{rr}-5,1 & 12,4\end{array}$ & Latvia & $51,9=84,4$ \\
\hline Armenia & $5,1,1$ & Russia & $51,7 \quad$ ln 6118 \\
\hline Albania & $4.9-12.4$ & Ukraine & $51,6-58,4$ \\
\hline Cyprus & $-3,8-1,2,4$ & Estonia & 50,3 l 62.5 \\
\hline Israel & 4.2 .20 & Azerbaijan & ab 1223 U MAL \\
\hline Azerbaijan & $-3.21,0$ & Turkey & 37.2. 10,9 \\
\hline Turkey & 1as & Ibekistan & $2.1 \mathrm{FEM}$ \\
\hline \multicolumn{2}{|c|}{ 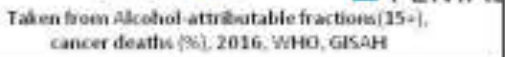 } & \multicolumn{2}{|c|}{ 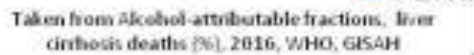 } \\
\hline
\end{tabular}

Figure 9. Alcohol-attributable fractions $(15+)$ cancer respectively cirrhosis death (\%) 
In Figure 9 we pointed the relation between gender and Alcohol-attributable fractions of death for cancer respectively cirrhosis. We can see that Romania occupies the position 3 in descending order for both of the characteristics previously mentioned. In addition, we can see that, especially for cancer death, the proportion that is attributable to alcohol is much greater for men than for women. We calculated the correlation between "Alcohol-attributable fractions cancer deaths" for men and the number indicating how many times alcohol-attributable fraction of cancer deaths is bigger for men than for woman and we obtained the value 0,107 , which is very small but indicates, however, an inverse relation between variables. When we did the same operations for ,Alcohol-attributable fractions liver cirrhosis deaths" we obtained a correlation coefficient of - 0,225 which shows, again, that gender gap regarding alcohol harm consequences tend to decrease when the incidence of the phenomenon increase. In figure 9 we can see, also, that, for countries ranked among the first (including Romania), almost the entire „Liver cirrhosis age-standardized death rates" is due to alcohol abuse. Regarding the death rate due to liver cirrhosis (per 100.000 people), Romania occupies the $8^{\text {th }}$ position in descending order of male ranking with a value of 51.8 (for women the death rate due to liver cirrhosis is only 22.9). The correlation between men death rate due to liver cirrhosis and the rapport between men and women death rates determined by the same disease is $-0,164$. The negative value shows that when death rate due to liver cirrhosis increase, gender gap tend to be smaller.

At the end of the presentation of data obtained we wish to reveal an important aspect: the relation between alcohol related variables which concerns men, respectively women. So, we could see that, apart from gender gap that is important for each aspect that we analyzed, there is a statistical tendency of simultaneously variation of values characterizing men and women.

From Table 1 we can see that correlations are very strong, close to 1 , for the variables describing male and female behavior regarding alcohol consumption (quantity, frequency and heavy episodic drinking). This means that linear formula „Y=aX+b" describe with good accuracy the relation between females' and males' variables targeting alcohol patterns of consumption. In other words, countries differ very much in the amount of alcohol drinking but they are quite similar in what concern the socially acceptable rapport between males' and females' consumption. Regarding harmful consequences of alcohol, correlations between men' and women' variables are not so high as those obtained in the case of consumption patterns but, however, an important part of the variable variance of one gender can be "explained" by the variable characteristic to the opposite gender.

\section{Review of theories and statistical European data}

Because our subject of interest was the differences between the two sexes regarding the patterns of alcohol consumption and the way in which this differences are reflected in drink harmful effects upon man and woman, we will remind some relevant data provide by WHO (GSRAH). In 2016 alcohol abuse caused 3 million deaths $(5.3 \%$ of all) from which 2.3 million were men, representing the overwhelming majority of alcohol victims. As an important result of drinking 
abuse, 237 million men and 46 million women experience alcohol use disorder (AUD) which means that the prevalence, for males, is 5.2 times bigger than for females.

With respect to the "Percentage of total deaths attributable to alcohol, by age group, 2016" for the category with highest risk (25-44 years) we can indicate that for men the proportion of death caused by alcohol lies between 31 and $29 \%$ while for women the range is $18-17 \%$ (approximate a half of the values characterizing men). Alcoholism is, again, much more frequent for men: $8,4 \%$ of them are alcohol addicted and $6,1 \%$ use it in harmful manner (women percent of alcoholics is only $1.5 \%$ - representing a 5,6 times lower value than for men - and only $2 \%$ among them are drinking in harmful way).

In Europe, zone with the lowest proportion of lifetime abstinence (of entire world) gender differences are quite important: $17.2 \%$ for male and $29.3 \%$ for female (of 1.7 times bigger than for men). Regarding pure alcohol consumption per capita, Europe is, again, on the first place with an average of 23.8 liters for men and 8.3 liters for women (having a 2.9 lower consumption than men). We must note, in addition, that prevalence of heavy episodic drinking is high in Europe (second place within world areas) and gender disparities are important: the prevalence is $56.5 \%$ for men and only $24.5 \%$ for women ( 2.3 times lower than for male). For teenagers, Europe occupies first place of the world regarding heavy episodic drinking, with a prevalence of $36.2 \%$ for boys and $11,5 \%$ for girls (3.1 times lower than for boys). We would like, now, to present a few answers to the question: why men consume more alcohol and in a much dangerous manner than women. The short review will target only the bibliography that we consulted because, as we already mentioned, we didn't have access to statistical data on the causality of gender disparities regarding alcohol consumption patterns. Bratberg et al. (2016) consider that relations from men and women alcohol consumption depend, on the same time, to biological particularities and specific gender roles culturally attributed within the society. Authors suggest, in addition, that general equality between sexes supposes more similarity in alcohol consumption patterns. The author reveals that despite all changing in gender roles, differences between sexes on alcohol patterns of consumption are still important and are influenced by a multitude of factors like age, education, job, civil status and the childcare. However, the nature of the influence changes from a society to another.

Lebreton (2016) suggests an explanation for gender disparities regarding alcohol patterns of consumption: the participation of young males to ,jock culture", described by sexism, admiration for muscular body, competitive spirit, heavy drinking episodes and rejection of intellectualism or homosexuality. To explain the gender gap in alcohol consumption it is relevant the author's conclusion from a field research from which results that the proportion of male who practice sports is higher than of women and their implication is more intense (expressed in hours of sports). So the participation of male and female at jock culture differs very much.

Foster et al (2015) refer to gender disparities in the prevalence of alcohol use disorder (AUD) and observe that these disparities determined the separate analysis of AUD risk factors and of their consequences for male and female. The author considers that using gender as an intermediate variable to support the explanation of the correlation between risk factor and the consequences of 
AUD, will allow making more clear the etiology and clinical results. Foster et al (2015) used an important sample which was traced between 17 and 29 years and it was tested regarding gender disparities for psychosocial risk factors and their outcomes in teenage and maturity, after the control of gender disparities in the base rates of AUD and in psychosocial dimensions. Psychosocial factors refers to the consumption of alcohol and other drugs, externalizing and internalizing symptoms, deviant peer association, family troubles, school difficulties, viewpoints and use of drugs by boy's or girl's friend, and socio-economic position as mature person. At maturity AUD consequences has been more important for females than for males and internalizing risk was more intense related with AUD for females (both at 17 and 29 years). The conclusion of the authors is that, in spite of more important average levels of risk for males, AUD is a more serious medical condition for females, which is distinguished by greater adolescent risk factors and higher level of AUD consequences by comparing to men. In addition, internalizing symptoms seem to be a risk factor for AUD especially for female.

Evans et al. (2017) intended to analyze on one hand, gender disparities in the relation between childhood adversities and various kinds of drugs use disorders and, on the other hand, to find out if gender is moderating this kind of relations. Authors used data from a large sample on men and women to establish if gender intermediate the correlation among childhood adversity and lifelong apparition of alcohol, drug and poly-substance related disorders. For every kind of substance use disorder, the risk is rising with the exposing at childhood adversity and females have a smaller risk than males. Nevertheless, moderation effects put into evidence that higher frequency of childhood adversity, gender disparities regarding predicted probability for a disorder became smaller with respect to alcohol, disappear in rapport to drugs, so that the risk within women group exceed that specific to men and, finally, gender gap widened in relation to poly-substances. The information about substance-specific gender disparities, correlated with knowledge about infancy adversity exposure, can help identify appropriate medical treatment.

Jalling et al .(2017) highlight the strong correlation between dangerous use of alcohol by teenagers and the perception of peers' behavior and, in addition, with the way of using own money and with some deviant conducts. The targets of authors study were to test short-term predictors of Heavy Episodic Drinking (HED) and the risk of alcohol consumption within a group of male and female being between 12 and 18 years, characterized by high externalizing and deviant conduct and alcohol consumption. As a conclusion, Jalling et al (2017) pointed that the peer group drinking perception represent valid predictors for both males' and females' HED and alcohol risk-use and, in addition, externalizing behavior may anticipate women dangerous use of alcohol. Parents' level of externalizing behavior represents a predictor only for the probability that their sons consume alcohol. As final observation, authors concluded that men and women have in common some predictors of dangerous consumption of alcohol and that perception of peer drinking seems to be one of most capable to anticipate the hazardous alcohol use.

The study of Zimmermann et al (2011) analyzed instrumental (masculine stereotyped) and expressive (feminine stereotyped) personality characteristics and alcohol consumption between male from Germany and Spain. It was used an 
sample of 76 German and 77 Spanish who's members were questioned about their alcohol consumption during daytime and nighttime and, in addition, they completed a Short Form of the Bem Sex Role Inventory. The characteristics evaluated with the Inventory and their interaction with the nationality was valid predictors for daytime and nighttime alcohol use. The findings sustain the hypothesis that alcohol consumption is related to the construction of male role and, in addition, the internalizing of traditional characteristic of female predict low health risk, as alcohol misuse.

After the review of the literature that we consulted considering the possible causes of the gender disparities on the patterns of alcohol consumption we are going to present our results on the issue which we were able to address, taking into account the data we had access to: gender disparities on drinking and their reflection in the prevalence of diseases related to alcohol.

\section{Discussion}

We remind the fact that the vast majority of the dates that we used (regarding year 2016) were provided by a database managed by World Health Organization who offers access to statistical information about the whole world, including the 53 European countries (more precisely, through the „Global Information System on Alcohol and Health" - GISAH). Only statistics from 2014 which refers to the frequency of alcohol consumption were obtained from EUROSTAT, targeting only EU countries and 3 others from Europe: Iceland, Norway and Turkey. We must remind, briefly, that Romania has important alcohol consumption $\left(8^{\text {th }}\right.$ place from 53 European countries), that women drink 4.2 times less than men (place 21 from 51 countries in descending order of men to women proportion). We must add, also, that in every country men drink more than women, beginning with Turkey, where the rapport is the highest 9.1 and finishing with Luxembourg, where the value is the lowest (3.1). Last but not least, we found a very strong relation between the total consumption of alcohol for men and for women and also, a strong negative correlation between total alcohol consumption and the number indicating how many times men drink more than women. We can say, on one hand, that women consume more alcohol if men do the same thing and, on the other hand, as the total consumption rise, differences between sexes tend to be lower.

Regarding the frequency of alcohol consumption, Romania occupies a low position both for the answers to "DAILY" and for "DAILY OR WEEKLY" consumption (place 19 respectively and 25 of 30 countries in descending order of responses percentages). In consensus, those who declare that they didn't drink „last month", „last year” or „never” are occupying the forth position in descending order of answers'. Data show that men consume much more frequent alcohol than women. With a value of 12 Romania occupies a second position (in descending order of the value indicating how many times is bigger the percent of men who drink daily comparing to woman' percent). The highest value is characteristic to Estonia (21) and the lowest, to United Kingdom (1.87). We find, again, the negative correlation between proportion of total daily drinkers and the ratio between the proportion of men and women who consume alcohol every day. We highlight, in 
addition, the very high correlation between the percents of male and female who consume alcohol daily.

Concerning abstainers (WHO 2016), Romania occupies the $25^{\text {th }}$ position from 51 countries and we found a strong negative coefficient of correlation between the total proportion of abstinent and the rapport between men and women share who never drink alcohol. We add that the correlation between abstainers males and females proportion is very high $\left(0,944^{* *}\right)$ and, also, that the proportion of abstinent women is 3.1 times bigger than that of men (place 22 of 51 in descending order of the proportions rapports).

The prevalence of heavy episodic drinking is relatively high in Romania that occupies the $12^{\text {th }}$ position (with 39\%) in descending order of the percent of persons who had such an episode past 30 days. Alcohol misuse is 1.84 times more frequent for men than for women and, according to this criterion, Romania is ranked on the $11^{\text {th }}$ position (on descending order of the values). We must add, again, that total percent of people who declare, past 30 days, at least a heavy episodic drinking is strongly correlated with the rapport between the proportion of men respectively of women being in the same situation. As a reiteration of last observations, the prevalence of „heavy episodic drinking” is strongly correlated between men and woman. We must note that the information obtained for the entire population regarding "heavy episodic drinking” are valid, also, for young people, having $15-19$ years.

With respect to alcohol addiction, Romania occupies place 47 from 51 in descending order of the percents of alcoholics specific to European countries. In our country the proportion of alcohol dependents is 3.3 times greater for men than for women and, at European level, there is no correlation between the total proportion of alcoholics and the rapport between men and women (as percents of addicted persons). And, again, like for the others variables, alcohol addiction prevalence is strongly correlated between the two genres $(\mathrm{r}=0,799)$.

Concerning to alcohol use disorder (AUD), our Country is ranked in the $47^{\text {th }}$ position of 51 by decreasing order of the proportions of people with AUD. Unexpectedly, we found a low but positive correlation between the proportion of people with AUD and the rapport between men' and women' proportion having that disorder (so, if AUD prevalence is greater, gender gap tend, also, to increase). As with the other variables, the percent of males having AUD is strongly correlated with the proportion of female having the same medical condition.

Concerning to „Alcohol-attributable fractions, all-cause deaths (\%)", Romania is placed on an unfavorable position (11 of 50 countries ranked in descending order). The correlation between the percent of these „Alcohol-attributable fractions" and the rapport of proportions characteristic for males, respectively for females is strongly negative $(\mathrm{r}=-0.794)$. The fraction from all-cause deaths that are attributable to alcohol correlates intensely between the two genders $(0,944)$.

Referring only at alcohol-attributable fractions of death for cancer respectively liver cirrhosis we find a much more worrying position 3 for both diseases (ranked in descending order of ,Alcohol-attributable fractions of death" for each illness). For both cancer and liver cirrhosis the correlation between ,the percent of alcohol-attributable fractions of death" and the rapport between men and women 
(characterized by the same fractions mentioned before) has low values but they are negative.

With respect to one of most dangerous consequence and more strongly related to alcohol abuse, liver cirrhosis (evaluated as ,age-standardized death rates”), Romania is ranked on the $8^{\text {th }}$ place in descending order of death rate.

The correlation between males death rate determined by liver cirrhosis and the rapport between male and female death rates (due to the same medical condition) is low but negative $(-0,164)$. We must add that correlation between the variables characterizing men and women liver cirrhosis death is strongly positive $(0,920)$.

In the end of our analysis we tried to synthesize statistical data presented until now (Table 1) and to add correlation between variables referring to the pattern of alcohol consumption and those evaluating its harm consequences (Table 2 and 3 ).

For Romania, the highest disparity between men and women was found for the frequency of daily alcohol consumption, our country occupying second position of 30 (by descending order of the rapport between men and women proportion of daily drinking).

Table 1.Statistical data on variables regarding alcohol and its consequences (by gender)

\begin{tabular}{|l|l|l|l|l|}
\hline $\begin{array}{l}\text { The issue that is subject } \\
\text { to attention }\end{array}$ & $\begin{array}{c}\text { Correlation } \\
\text { between Men } \\
\text { and Women }\end{array}$ & $\begin{array}{c}\text { Place by the } \\
\text { total variable }\end{array}$ & $\begin{array}{c}\text { Men re- } \\
\text { ported to } \\
\text { women }\end{array}$ & $\begin{array}{c}\text { Correlation be- } \\
\text { tween variable and } \\
\text { the rapport Men/ } \\
\text { Women }\end{array}$ \\
\hline $\begin{array}{l}\text { Alcohol, total per capita } \\
(15+) \text { consumption (in } \\
\text { liters of pure alcohol) }\end{array}$ & $0.953^{* *}$ & 8 of 53 & $\begin{array}{c}4.2 \\
21^{\text {th }} \text { place }\end{array}$ & $-0.804^{* *}$ \\
\hline $\begin{array}{l}\text { Frequency of alcohol } \\
\text { consumption by sex } \\
\text { DAlLY }\end{array}$ & $0.903^{* *}$ & 19 of 30 & $\begin{array}{c}12 \\
2^{\text {th }} \text { Place }\end{array}$ & $-0.435^{*}$ \\
\hline $\begin{array}{l}\text { Frequency of alcohol } \\
\text { consumption by sex } \\
\text { DAlLY or WEEKLY }\end{array}$ & $0.855^{* *}$ & 25 of 30 & $\begin{array}{c}6.09 \\
2^{\text {th }} \text { place }\end{array}$ & $-0.714^{* *}$ \\
\hline $\begin{array}{l}\text { Never consume alcohol } \\
\text { last MONTH/YEAR or } \\
\text { never }\end{array}$ & $0,846^{* *}$ & 4 of 30 & $\begin{array}{c}0.37 \\
10^{\text {th }} \text { place }\end{array}$ & +0.221 \\
\hline Abstainers & $0,944^{* *}$ & 25 of 51 & $\begin{array}{c}0,33 \\
28^{\text {th }} \text { place }\end{array}$ & $+0.727^{* *}$ \\
\hline $\begin{array}{l}\text { Alcohol, heavy episodic } \\
\text { drinking (15+) past 30 } \\
\text { days (\%) }\end{array}$ & $0.939^{* *}$ & 12 of 51 & $\begin{array}{c}2.7 \\
40^{\text {th }} \text { place }\end{array}$ & $-0.822^{* *}$ \\
\hline $\begin{array}{l}\text { Heavy episodic drinking } \\
\text { (youth 15 -19 years) past } \\
\text { 30 days (\%) }\end{array}$ & $0,935^{* *}$ & 14 of 51 & $\begin{array}{c}3.1 \\
38^{\text {th }} \text { place }\end{array}$ & $-0.810^{* *}$ \\
\hline
\end{tabular}




\begin{tabular}{|l|l|l|c|l|}
\hline $\begin{array}{l}\text { Alcohol dependence } \\
\text { prevalence } 1 \text { year, age } \\
15 \text { years+ }\end{array}$ & $0,799^{* *}$ & 47 of 51 & $\begin{array}{c}3.3 \\
40^{\text {th }} \text { place }\end{array}$ & 0.00 \\
\hline $\begin{array}{l}\text { Alcohol use disorders } \\
(15+), 12 \text { month preva- } \\
\text { lence }(\%)\end{array}$ & $0,797^{* *}$ & 47 of 51 & $\begin{array}{c}3.2 \\
41^{\text {th }} \text { place }\end{array}$ & +0.227 \\
\hline $\begin{array}{l}\text { Alcohol-attributable frac- } \\
\text { tions, all-cause deaths } \\
(\%)\end{array}$ & $0,944^{* *}$ & 11 of 51 & $\begin{array}{c}2,6 \\
37^{\text {th }}\end{array}$ place & $-0.794^{* *}$ \\
\hline $\begin{array}{l}\text { Alcohol-attributable } \\
\text { fractions (15+), cancer } \\
\text { deaths }\end{array}$ & $0,485^{* *}$ & 3 of 51 & $\begin{array}{c}2.5 \\
26^{\text {th }} \text { place }\end{array}$ & $\begin{array}{l}-0.107 \\
\text { (total replace with } \\
\text { men) }\end{array}$ \\
\hline $\begin{array}{l}\text { Alcohol-attributable frac- } \\
\text { tions (15+), liver cirrhosis } \\
\text { deaths }\end{array}$ & $0,761^{* *}$ & 3 of 51 & $\begin{array}{c}1.3 \\
23^{\text {th }} \text { place }\end{array}$ & $\begin{array}{l}-0,225 \\
\text { (total replace with } \\
\text { men) }\end{array}$ \\
\hline $\begin{array}{l}\text { Liver cirrhosis, } \\
\text { age-standardized death } \\
\text { rates (15+), per } 100,000\end{array}$ & $0,920^{* *}$ & 8 of 51 & $\begin{array}{c}2.3 \\
29^{\text {th }} \text { place }\end{array}$ & $\begin{array}{l}-0.164 \\
\text { (total replace with } \\
\text { men) }\end{array}$ \\
\hline
\end{tabular}

Data calculated using information from EUROSTAT 2014 and WHO-GISAH 2016

\section{${ }^{* *}$ Probability greater than 0.99 and * Probability greater than 0.95}

In first place, we must reiterate the important observation that, even there are large gender disparities between the quantities or frequencies (including heavy episodic drinking) of alcohol consumption in each country, at European level, there is an extremely high correlation between the variables corresponding to men and women. This fact suggest that there is a good concordance between the ordering of European countries by variables which characterizes men', respectively women' alcohol use. We can see (Table 1) that observations made for genders alcohol consumption are valid, also, for the harmful consequences of men and women drinking. We can assume that cultural specific patterns lie behind the men and women habit of alcohol consumption in each European country and that these habits are reflected in the different harmful effect of drinking for the two sexes.

Concerning Romania's position within European countries, data are not easy to understand: alcohol consumption per capita is relatively high but its frequency of consumption is (very) low and the proportion of abstainers is moderate. In what concern harmful alcohol consumption, Romanians practice relatively frequent ,heavy episodic drinking", both for men and women. With respect to harmful consequences of alcohol abuse, Romania hold a very low prevalence of alcoholism and AUD but, in opposition, alcohol consumption has an important contribution to deaths rate (determined by all-cause, by cancer respectively by liver cirrhosis) and, also, our country is characterized by a large liver cirrhosis deaths rate. From Table 1 we can observe that, for alcohol quantity, frequency and heavy episodic drinking, the correlation between these characteristics and the variables defined as the rapport between males and females is strongly negative, indicating that gender gap decrease 
as alcohol consumption increase. We must add that for variables indicating abstinence or low frequency of alcohol consumption (for which the ratio of men and women is subunit), the global rise of alcohol low consumers correlates with the decrease of gender gap. Regarding harmful consequences of alcohol consumption, only for "alcohol-attributable fractions, all-cause deaths (\%)" is valid the observation that we made before (for the others variables reflecting harmful alcohol consequences the correlation coefficient is negative, except AUD, but of low level).

In Table 1 it was highlighted the value of the rapport between variables related to men and women (referring to alcohol use and its consequences). For Romania, as for the others European country, every dimension that we considered put women in a better position than men (they consume lower quantities, less frequent and less hazardous, with reduced negative consequences). Further, we will present the correlations between, on one hand, the variables representing the amount of alcohol consumption, the proportion of abstainers and the frequency of binge drinking and, on other hand, the characteristics which describe harmful consequences of drinking (separately for each gender). First we must highlight that variables referring to the patterns of alcohol consumption are strongly correlated one to another both for women and for men with values between 0,988 and 0,743. All values are positive with the exception of the correlations between the proportion of abstainers and the other aspects of alcohol consumption, which are, obviously, negative. It means, statistically, the higher the consumption of alcohol, the more its frequency increase, simultaneously with ,binge drinking” prevalence and with the decrease of abstainers' proportion.

Table 2. Correlations between male variables on alcohol use and its

\begin{tabular}{|c|c|c|c|c|}
\hline & $\begin{array}{l}\text { Male Total } \\
\text { consumption } \\
\text { of pure alco- } \\
\text { hol (L) }\end{array}$ & $\begin{array}{c}\text { Male } \\
\text { Alcohol, } \\
\text { abstai- } \\
\text { ners } \\
\text { lifetime } \\
\text { (\%) }\end{array}$ & $\begin{array}{c}\text { Male Alcohol, } \\
\text { heavy episodic } \\
\text { drinking }(15+) \\
\text { past } 30 \text { days } \\
\text { (\%), Entire } \\
\text { population }\end{array}$ & $\begin{array}{c}\text { Male Heavy episodic } \\
\text { drinking (youth } 15 \\
-19 \text { years) past } 30 \\
\text { days (\%) }\end{array}$ \\
\hline $\begin{array}{l}\text { Male Total consumption } \\
\text { of pure alcohol (liters) }\end{array}$ & 1 &,$- 743 * *$ &, $868 * *$ &, $841 * *$ \\
\hline $\begin{array}{l}\text { Male Alcohol, abstai- } \\
\text { ners lifetime (\%) }\end{array}$ &,$- 743^{* *}$ & 1 &,$- 835^{* *}$ &,$- 828 * *$ \\
\hline $\begin{array}{l}\text { Male Alcohol, heavy } \\
\text { episodic drinking }(15+) \\
\text { past } 30 \text { days (\%), Total }\end{array}$ &, $868 * *$ &,$- 835 * *$ & 1 &, $988 * *$ \\
\hline $\begin{array}{l}\text { Male Heavy episodic } \\
\text { drinking (youth } 15-19 \\
\text { years) past } 30 \text { days (\%) }\end{array}$ &, $841^{* *}$ &,$- 828 * *$ &, $988 * *$ & 1 \\
\hline $\begin{array}{l}\text { Male Alcohol depen- } \\
\text { dence prevalence } 1 \\
\text { year, age } 15 \text { years+ }\end{array}$ & ,316* & -177 & ,336* & ,279* \\
\hline
\end{tabular}




\begin{tabular}{|l|l|l|c|c|}
\hline $\begin{array}{l}\text { Male Alcohol use disor- } \\
\text { ders (15+), 12 month } \\
\text { prevalence (\%) }\end{array}$ &, $385^{* *}$ &,- 187 &, $397^{* *}$ &, $336^{*}$ \\
\hline $\begin{array}{l}\text { Male Alcohol-attributa- } \\
\text { ble fractions, all-cause } \\
\text { deaths (\%) }\end{array}$ &, $431^{* *}$ &,- 159 &, 232 &, 157 \\
\hline $\begin{array}{l}\text { MALE Alcohol-attribu- } \\
\text { table fractions (15+), } \\
\text { cancer deaths }\end{array}$ &, $597^{* *}$ &,$- 404^{* *}$ &, $372^{* *}$ &, $310^{*}$ \\
\hline $\begin{array}{l}\text { Male Alcohol-attributa- } \\
\text { ble fractions (15+), liver } \\
\text { cirrhosis deaths }\end{array}$ &, $446^{* *}$ &,$- 540^{* *}$ &, $411^{* *}$ &, $411^{* *}$ \\
\hline $\begin{array}{l}\text { MALE Liver cirrhosis, } \\
\text { age-standardized death } \\
\text { rates (15+), per 100,000 } \\
\text { populatio }\end{array}$ &,- 045 &, 272 &,$- 334^{*}$ &,$- 402^{* *}$ \\
\hline
\end{tabular}

Calculated based on Global Information System on Alcohol and Health, WHO

Table 3. Correlations between female variables on alcohol use and its consequences

\begin{tabular}{|l|l|l|l|l|}
\hline & $\begin{array}{l}\text { Female } \\
\text { Tot, con- } \\
\text { sumption } \\
\text { of pure } \\
\text { alcohol (L) }\end{array}$ & $\begin{array}{l}\text { Female } \\
\text { Alcohol, } \\
\text { abstainers } \\
\text { lifetime } \\
(\%)\end{array}$ & $\begin{array}{l}\text { Female Alcohol, } \\
\text { heavy episodic } \\
\text { drinking (15+) } \\
\text { past 30 days (\%), } \\
\text { Entire pop. }\end{array}$ & $\begin{array}{l}\text { Female Heavy } \\
\text { episodic drin- } \\
\text { king (youth 15 } \\
-19 \text { years) past } \\
30 \text { days (\%) }\end{array}$ \\
\hline $\begin{array}{l}\text { Female Total consumption } \\
\text { of pure alcohol (liters) }\end{array}$ & 1 &,$- 875^{* *}$ &, $884^{* *}$ &, $872^{* *}$ \\
\hline $\begin{array}{l}\text { Female Alcohol, abstainers } \\
\text { lifetime (\%) }\end{array}$ &,$- 875^{* *}$ & 1 &,$- 763^{* *}$ &,$- 766^{* *}$ \\
\hline $\begin{array}{l}\text { Female Alcohol, heavy epi- } \\
\text { sodic drinking (15+) past } \\
30 \text { days (\%), Total }\end{array}$ &, $884^{* *}$ &,$- 763^{* *}$ & 1 &, 984 \\
\hline $\begin{array}{l}\text { Female Heavy episodic } \\
\text { drinking (youth 15 -19 } \\
\text { years) past 30 days (\%) }\end{array}$ &, $872^{* *}$ &,$- 766^{* *}$ &, $984^{* *}$ & 1 \\
\hline $\begin{array}{l}\text { Female Alcohol dependen- } \\
\text { ce prevalence 1 year, age } \\
15 \text { years+ }\end{array}$ &, $321^{*}$ &,$- 289^{*}$ &, $333^{*}$ &, $300^{*}$ \\
\hline $\begin{array}{l}\text { Female Alcohol use di- } \\
\text { sorders (15+), 12 month } \\
\text { prevalence (\%) }\end{array}$ &, $466^{* *}$ &,$- 402^{* *}$ &, $473^{* *}$ &, $434^{* *}$ \\
\hline
\end{tabular}




\begin{tabular}{|l|l|l|l|l|}
\hline $\begin{array}{l}\text { Female Alcohol-attribu- } \\
\text { table fractions, all-cause } \\
\text { deaths (\%) }\end{array}$ &, $398^{* *}$ &,- 218 &, $366^{* *}$ &, $302^{*}$ \\
\hline $\begin{array}{l}\text { FEMALE Alcohol-attributa- } \\
\text { ble fractions (15+), cancer } \\
\text { deaths }\end{array}$ &, $837^{* *}$ &,$- 694^{* *}$ &, $619 * *$ &, $581^{* *}$ \\
\hline $\begin{array}{l}\text { Female Alcohol-attributa- } \\
\text { ble fractions (15+), liver } \\
\text { cirrhosis deaths }\end{array}$ &, $950^{* *}$ &,$- 955^{* *}$ &, $830^{* *}$ &, $815^{* *}$ \\
\hline $\begin{array}{l}\text { FEMALE Liver cirrhosis, } \\
\text { age-standardized death } \\
\text { rates (15+), per 100,000 } \\
\text { populatio }\end{array}$ &,- 232 &, $398^{* *}$ &,$- 314^{*}$ &,$- 334^{*}$ \\
\hline
\end{tabular}

\section{Calculated based on Global Information System on Alcohol and Health, WHO}

Total consumption of pure alcohol correlates positively with alcohol dependence prevalence, both for men and women, with AUD and with alcohol- attributable fraction within: ,all cause death”, „,ancer death” respectively, „liver cirrhosis deaths". For women, comparing to men, correlation coefficient is stronger between total alcohol consumption and alcohol-attributable fractions to "cancer deaths" respectively to „liver cirrhosis deaths”. In what concern „liver cirrhosis death rates" correlation, for males, is null and for females is slightly negative. This apparent paradoxe may be explained by interaction between variables such as: countries with higher alcohol consumption having, at the same time, verry good health care.

The proportion of abstainers correlates negatively with all harmful effects of alcohol consumption, except „liver cirrhosis death rate" with which the correlation is positive for both genders (probably for liver cirrhosis there are country characteristics, such as the prevention of risk factors, good quality medical services, and support post-hospitalization that may be more relevant that cultural patterns of alcohol consumption). We must add that for all negative effects of alcohol consumption, correlations with ,liver cirrhosis death rate” are stronger for females than for males.

The prevalence of ,Alcohol heavy episodic drinking” correlates positively with all negative effects of alcohol misuse and, again, for females the connection is stronger than for males. This observations are valide both for the entire population (age 15+) and for youngers (15-19 years). 


\section{Conclusion}

In the whole World and, in particular, in Europe men are much more affected by alcohol harmful effects than women and the explanation seems to be very simple: in each country females have moderate patterns of drinking comparing to males. Theories reviewed suggest some explanations for alcohol consumption gender gap: differences in gender roles attributed to men and women but, also, biologic particularities or social background characteristics: age, education, job, civil status and childcare. There are cultural theories that assume sexism and hedonism as the base of gender disparities regarding alcohol consumption. Other researches reveal that men have higher psycho-social risk for AUD but women are more vulnerable at health alcohol consequences. Gender differences in alcohol consumption may be explained by males' higher risk of exposing at childhood adversity (comparing to females) which increase the probability of substance abuse, including alcohol. The disparity between alcohol consumption of boys and girls (adolescents) can, also, be linked with the different way in which they perceive the behavior of peer group (related, especially, to alcohol misuse). We mention, as an important hypothesis, the idea that gender differences regarding alcohol drinking depends on the level of internalizing for traditional personality dimensions: instrumental (masculine stereotyped) and expressive (feminine stereotyped).

So, cultural and biological differences combined with particular life experiences are directly related to gender disparities in alcohol pattern of consumption. We will concentrate, now, on the problem that we tried to make clearer, using statistical data that we had: which are the differences in alcohol consumption between men and women and how are reflected its negative consequences on each gender, separately (at EU level and in Romania, in particular).

Comparing Romania with European countries according to the patterns of alcohol consumption we note that results do not seems to be consistent: in our country alcohol consumption is higher that UE average level but the frequency of this behavior is relatively low and the percent of abstainers is moderate. The explanation may be the presence of a group of heavy episodic drinkers, not necessarily alcoholics but who are subject to harmful action of alcohol misuse.

In what concern the consequences of alcohol abuse, Romania has a low prevalence of alcoholism but is on the first places regarding the fraction of death rate attributable to alcohol (for all deaths, for those due to cancer, respectively to liver cirrhosis). In addition, death rate due to liver cirrhosis is one of highest in EU.

Data that we have do not permit a clear explanation for these inadvertences but we can assume that the structure of health care system and the accuracy on which are identified patients with alcohol (related) problems has a significant contribution. Certainly, the statistics concerning death rate approximates good the reality but data related to alcoholism and AUD (which are „shameful" diseases) is likely to be much underestimated (by example, a lot of alcoholics may prefer not to see a doctor or, at least, not to be registered as a patient).

Regarding European countries we must highlight that both for variables referring to patterns of alcohol consumption and for those concerning consequences of excessive drinking, correlations between values characteristic to men and women 
are very strong. This fact has to be taken into account together with the important observation that in each European country women have better positions than men regarding alcohol problems. So, we assume that social and cultural norms which sustain that woman must be more moderate that man on alcohol consumption lead to similar hierarchy for European countries depending on variables characteristic to each of two genders. The same mechanism it is probable to justify gender gap and gender correlation for the consequences of alcohol misuse (if women' alcohol consumption is moderate, it is normal that negative consequences are, proportionally, lower).

For variables defining high quantities and frequencies of alcohol consumption, we found that the rapport between men and women become smaller as much as the variable have a higher value. This means, for example, that as the consumption of alcohol per capita increases, the differences between men and women will be smaller. We can assume that if a behavior becomes more usual, social prescription regarding gender differences will lose their power. This statistical regularity should be reflected, also, upon the variables that describe consequences of alcohol consumption (for total population and separately, by sexes). As an exception, the correlation between alcohol dependence prevalence and the rapport between values characteristic to males respective to females we obtained a null result and for AUD the same statistical procedure leads even to a positive result. For the other variables designating alcohol misuse consequences, the correlations between their level and the rapport between the values characteristic to men and to women are negative but low (with exception for ,alcohol-attributable fractions, all-cause deaths", where correlation is strongly negative). It seems that the tendency of reducing differences between men and women when is increasing the prevalence of an alcohol related disease is unclear, probably because here the psycho-social factors do not have an important contribution or because there are a lot of variables that may mediate differentially (depending on sex) the relation between alcohol misuse and its consequences (by example, risk factors of alcohol related disease can differ by gender).

Finally, we can say that the different forms of alcohol misuse represent important predictors for alcohol related illnesses but the values of correlation coefficients vary very much from a couple of variables to another and, also, between masculine and feminine population. As an example, women' total alcohol consumption correlates very strong with alcohol-attributable fractions regarding cancer deaths and liver cirrhosis. For men, the same relation is much weaker. In addition, both for men and women, correlations of variables describing patterns alcohol consumption correlate stronger with alcohol-attributable fractions for cancer and liver cirrhosis deaths than with AUD or with alcoholism prevalence.

As the statistic data shows, women have a more healthy behavior on alcohol consumption than men but, also, their exposure to alcohol misuse has more serious consequences. Regarding our country we must highlight that socially most visible aspects, as being alcohol consumption per capita and the alcohol-attributable fractions of death (for all cause, for cancer respectively for liver cirrhosis), have high values comparing to European Countries. This fact makes Romania vulnerable to alcohol harmful consequences, especially because the results of alcohol exposure depends on socio-cultural variables, 
probably most important being gender roles together with the structure and quality of medical care system.

\section{References}

Bratberg, G., H., Wilsnack, S., C., Wilsnack, R., Haugland S., H., Krokstad, S., Sund, E., R.,\& Bjørngaard, J., H. (2016). Gender differences and gender convergence in alcohol use over the past three decades (1984-2008), The HUNTStudy, Norway, BMC Public Health, 16, 723.

EUROSTAT Database, https://ec.europa.eu/eurostat/data/browse-statistics-by-theme

Evans, E.A., Grella, C.E., Upchurch, D.M., (2017). Gender differences in the effects of childhood adversity on alcohol, drug, and polysubstance-related disorders. Social Psychiatry and Psychiatric Epidemiology, 52, 901-912.

Foster, K., T., Hicks, B., M., Iacono, W., G.,McGue, M. (2015). Gender differences in the structure of risk for alcohol use disorder in adolescence andyoung adulthood. Psychological Medicine, 45, 3047-3058.

Jalling, C., Elgán, T., H.,, Tengström, A., \& Birgegard A. (2017). Gender-specific predictors of at-risk adolescents' hazardous alcohol use-a cohort study. Substance Abuse Treatment, Prevention, and Policy, 12, 23.

Lebreton, F., Peralta, R., L., Allen-Collinson, J., Chervenak Wiley, L., Routier, G. (2017). Contextualizing Students' Alcohol Use Perceptions and Practices within French Culture: An Analysis of Gender and Drinking among Sport Science College Students. Sex Roles, 76, 218-235.

Sparkes, Andrew, Brown, David H. K. \& Partington, E. (2010). The "Jock, Body" and the social construction of space: the performance and positioning of cultural identity. Space and Culture, 13(3), 333-347.

WHO (2017). Global Information System on Alcohol and Health (GISAH), World Health Organization https://www.who.int/substance_abuse/activities/gisah/en/

WHO (2018). Global status report on alcohol and health 2018, Geneva: World Health Organization (WHO), 2018. License: CC BY-NC-SA 3.0, IGO, ISBN 978-92-4-156563-9,https://apps.who.int/iris/bitstream/handle/10665/ 274603/9789241565639-eng.pdf?ua=1

Zimmermann, F., Sieverding M., \& Muller, S.M. (2011). Gender-Related Traits as Predictors of Alcohol Use in Male German and Spanish University Students. Sex Roles, 64, 394-404. 